$$
\text { DOE/ER/40437-T2-PH, } 2
$$

PROPOSAL FOR THE RENEWAL OF DE-FGO5-88-ER40437 FOR THE PERIOD OF APRIL 1,1995 - MARCH 31, 1997, THE FIRST YEAR OF THE THREE YEAR CONTRACT APRII 1, 1995 - MARCH 31,1998

\title{
Pion and Kaon Correlations In High Energy Heavy-Ion Collisions
}

\author{
Kevin L. Wolf \\ Cyclotron Institute \\ Texas A\&M University \\ College station, $\mathrm{TX} 77843$
}

\section{SUMMARY OF PROPOSED TECHNICAL SCOPE}

Emphasis is placed on the trigger functions of experiments in the NA44 collaboration in preparation for further running of lead beams at the CERN SPS. A successful upgrade of the time-of-flight trigger electronics has established the feasibility and need for similar upgrades on other hodoscopes. A new impact parameter selection trigger counter has been designed and built and will be installed. Data analysis will continue on single particle spectra collected for $160 \mathrm{~A} \mathrm{GeV}$ lead and $450 \mathrm{GeV}$ proton collisions. It is planned to expand data analysis efforts into identical kaon interferometry. New experiments at decreased bombarding energies are proposed in the final year of this grant.

Analysis of data from the EOS time projection chamber will be completed with regard to two pion interferometry at Bevalac energies. The interpretation of this work is expected to be completed within the first six months of this grant.

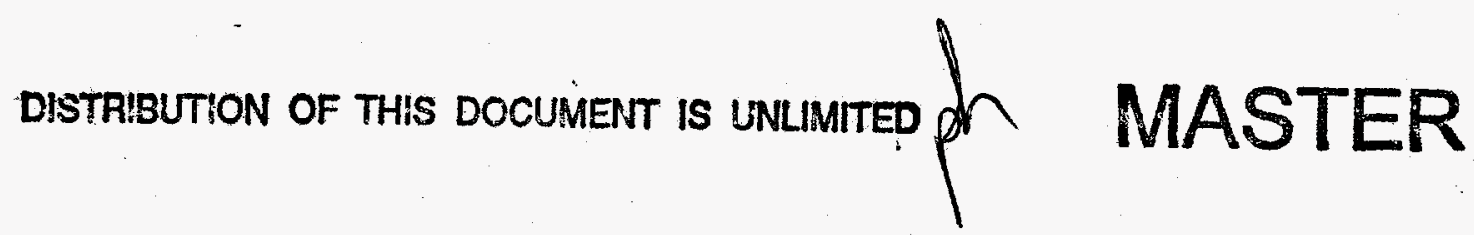




\section{DISCLAIMER}

This report was prepared as an account of work sponsored by an agency of the United States Government. Neither the United States Government nor any agency thereof, nor any of their employees, makes any warranty, express or implied, of assumes any legal liability or responsibility for the accuracy, completeness, or usefulness of any information, apparatus, product, or process disclosed, or represents that its use would not infringe privately owned rights. Reference herein to any specific commercial product, process, or service by trade name, trademark, manuiacturer, or otherwise does not necessarily constitute or imply its endorsement, recommendation, or favoring by the United States Government or any agency thereof. The views and opinions of authors expressed herein do not necessarily state or reflect those of the United States Government or any agency thereof. 


\section{DISCLAIMER}

Portions of this document may be illegible electronic image products. Images are produced from the best available original document. 
PROPOSAL FOR THE RENEWAL OF CONTRACT DE-FG05-88ER40437 FOR THE PERIOD FROM APRIL 1, 1995 - MARCH 31, 1998

First year of the three year period, April 1, 1995 - March 31,1996

ABSTRACT

Data analysis is in progress for recent experiments performed by the NA44 collaboration with the first running of $160 \mathrm{~A} \mathrm{GeV} 208 \mathrm{~Pb}$-induced reactions at the CERN SPS. Identified singles spectra were taken for pions, kaons, protons, deuterons, antiprotons and antideuterons. Twopion interferometry measurements were made for semi-central-triggered ${ }^{208} \mathrm{~Pb}+\mathrm{Pb}$ collisions. An upgraded multi-particle spectrometer allows high statistics data sets of identified particles to be collected near mid-rapidity. A second series of experiments will be performed in the fall of 1995 with more emphasis on identical kaon interferometry and on the measurement of rare particle spectra and correlations. Modest instrumentation upgrades by TAMU are designed to increase the trigger function for better impact parameter selection and improved collection efficiency of valid events. An effort to achieve the highest degree of projectile-target stopping is outlined and it is argued that an excitation function on the SPS is needed to better understand reaction mechanisms.

Analysis of experimental results is in the final stages at LBL in the EOS collaboration for two-pion interferometry in the $1.2 \mathrm{~A} \mathrm{GeV} \mathrm{Au+Au}$ reaction, taken with full event characterization. 


\title{
Grant DE-FG05-88ER40437 United States Department of Energy office of High Energy and Nuclear Physics
}

\author{
PROGRESS IN RESEARCH 1994
}

PION CORRELATIONS AND CALORIMETER DESIGN

FOR HIGH ENERGY HEAVY ION COLLISIONS

Kevin L. Wolf

Cyclotron Institute Texas A\&M University

College station, Texas 77843 


\section{PION CORRELATIONS AND CALORIMETER DESIGN FOR HIGH ENERGY HEAVY ION COLLISIONS}

TABLE OF CONTENTS

INTRODUCTION

page 1

I. THE EOS TIME PROJECTION CHAMBER

2

II. SAMPLING CALORIMETER CONSTRUCTION AND TESTS

22

III. EXPERIMENT NA44 AT CERN

IV. RECENT PUBLICATIONS

\section{RESEARCH STAFF}

K.L. Wolf, Professor A.D. Chacon, Research Associate; M. Murray, Research Associate; R.K. Choudhury, Visiting Scientist; H. Jabs, Graduate student; B. Sadhwani, Computer Systems Manager, M. Hamelin, Graduate student; J. Newsom, Graduate Student; M. Hodapp, Design Engineer 
I. INTRODUCTION

The analysis effort continued on EOS time projection chamber data at LBL during the past year. The TAMU interest in the effort is focussed on two-pion interferometry measurements in the TPC. The main goal of our work is to extract pion correlations relative to the reaction plane using full event reconstruction. Besides checking the effect of the hadronic matter on the correlation results, a secondary goal lies in the extraction of correlation parameters in a large solid angle device for comparision with results obtained previously from small solid angle spectrometers. In particular, a confirmation of the apparent oblate source shapes seen in central collisions would provide a benchmark that theoretical calculations must meet in the description of the space-time extent of the reaction zone at Bevalac and SIS energies.

An electromagnetic sampling calorimeter test section that features a highly developed optical fiber readout has been constructed in a continuation of our calorimeter development started for the Participant Calorimeter project for BNL. The 100-tower structure was constructed as a cost effective device optimized in segmentation and energy resolution for a high multiplicity environment expected at RHIC and at the LHC, and for good particle identification. The calorimeter was tested at the CERN PS with pions, muons and electrons in two modes. The design allows optical fibers to be changed for different requirements, as demonstrated in the tests. In high energy resolution mode, all fibers read out the full depth of the calorimeter ( 70 plates). In a second run at the PS the device was segmented four-fold longitudinally with the use of fibers with light blockage at the desired depths, to test the particle identification capabilities.

Development work has been performed for Na44 on trigger electonics which was used for the CERN lead beam upgrade, and $a$ new trigger counter was constructed for impact parameter selection in those experiments. The analysis of previously collected single -particle inclusive data has been completed for $450 \mathrm{GeV}$ proton-induced and $200 \mathrm{~A}$ GeV sulfur-induced reactions for pion, kaon and proton spectra. Data have been collected for the $160 \mathrm{~A} \mathrm{GeV} \mathrm{Pb}+\mathrm{Pb}$ system for proton, kaon and pion singles, and for two-pion correlations. 


\section{EOS TIME PROJECTION CHAMBER DATA ANALYSIS}

Simulations for two pion correlation measurements in the EOS TPC were reported previously in the 1992 progress report, where it was found to be quite promising in terms of two-track resolution and efficiency. A diagram of the experimental apparatus is shown in Fig.1. Before data analysis was started, it was believed that the major problem with a high acceptance device like the present TPC was in the collection of sufficient numbers of close correlated pion pairs to define the source parameters. Optimized small acceptance angle multiparticle spectrometers usually have a considerable advantage in that regard, and several sets of such high statistics data have been taken for heavy-ion reactions at LBL, BNL and CERN. Two sets of EOS data are believed to have sufficient statistics to provide good three dimensional pion source parameters, each of more than 30,000 close correlated pairs. Both cases represent the highest energies available at the Bevalac in the respective mass ranges, namely $1.9 \mathrm{~A} \mathrm{GeV} 58 \mathrm{Ni}+$ $\mathrm{Cu}$ and $1.2 \mathrm{~A} \mathrm{GeV} \mathrm{Au}+\mathrm{Au}$. The TAMU analysis effort for pion correlations has thus far been focused on central trigger $1.2 \mathrm{~A} \mathrm{GeV} \mathrm{Au}$ $+\mathrm{Au}$, which was a high statistics central trigger set, taken for the HBT work. Analysis of the data produced a rather unexpected result of a small source size for the large Au+Au system. A comparision of this analysis with systematics from other studies is shown in Fig.2 and is discussed in the next section by A.D. Chacon. The rather unsystematic result is attributed at this time to the tendency of the tracker routine used and of the TPC itself to split single tracks and make sets of false two or three close correlated tracks. Invalid close pairs generated in this fashion had to be eliminated to provide meaningful HBT results. The negative pion multiplicities extracted for $1.2 \mathrm{~A} \mathrm{GeV} \mathrm{Au}+\mathrm{Au}$ is low by a factor of two or more in comparision to systematics, which may be a result of attempting to track mips in a TPC that has been set up for a wide dynamic range of ionization densities. While these two problems are of high importance only for the pion HBT work in the present Bevalac data, both are of extreme importance for future experiments in $\mathrm{E895}$ at the AGS where pion production has a much higher probability. 


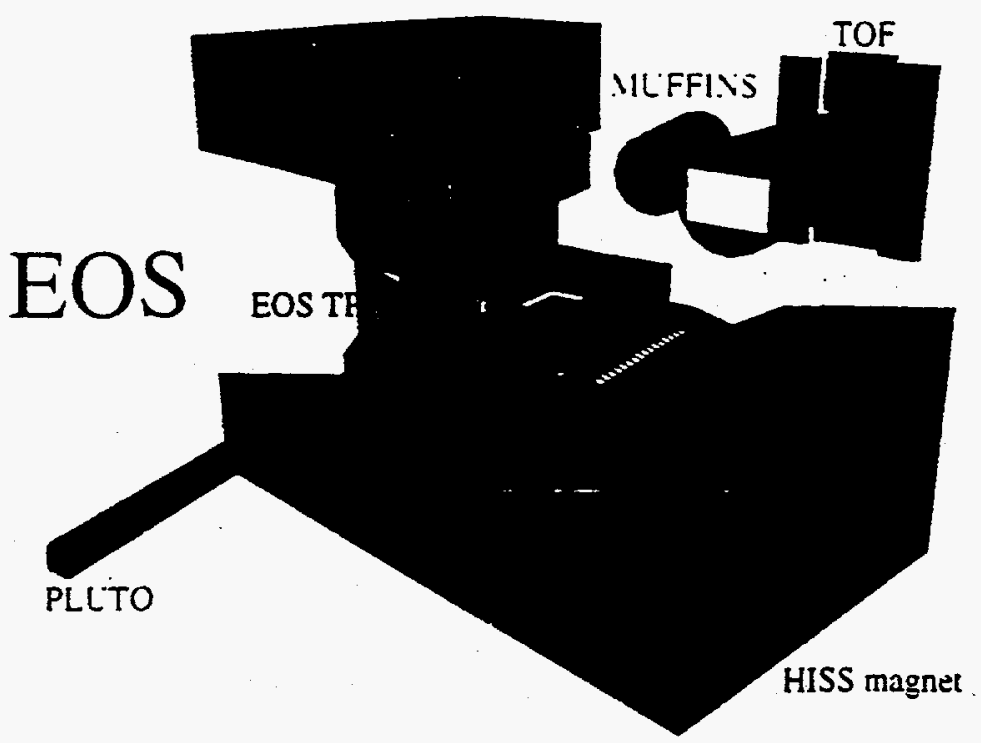

Figure 1 - Diagram of the EOS Time Projection Chamber and Detectors

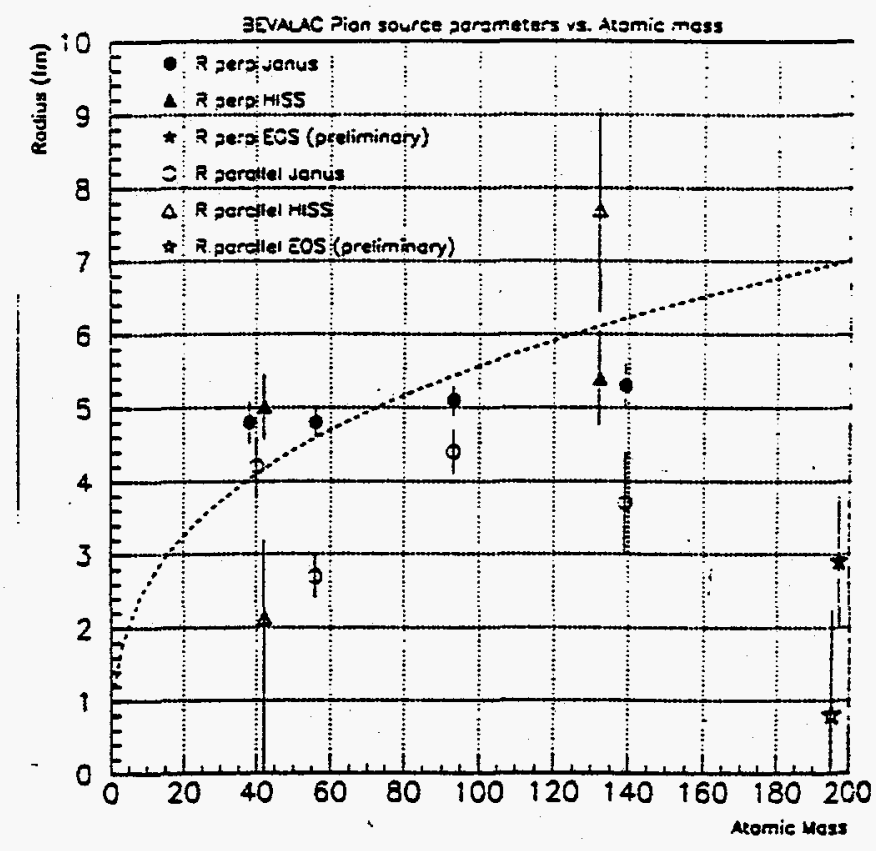

Figure 2 - A compilation of $\mathrm{HBT}$-derived radius parameters determined for several symmetric systems at Bevalac energies. 


\section{Track finding Studies in the EOS Time Projection Cham- ber}

This work was undertaken to improve the track finding software for the EOS Time Projection Chamber (TPC). The efficiency for finding pion pairs was initially about $39 \%$, which is quite low, and it was hoped that the efficiency could be improved. In the EOS TPC, ionizing particles from a nuclear collision ionize the TPC gas, and the liberated electrons are drifted by electric and magnetic fields to the pad plane structure, where the number of electrons is amplified in the gas, and a signal is produced in the capacitivelycoupled pad plane. The location of the signal on the pad plane locates the projection of the ionization onto the pad plane. From the strength and time of the signals on the pad plane, the amount of ionization and the drift time are deduced. The drift time, coupled with the drift speed, gives the height of the original ionization above the pad plane. This information allows a 3-dimension reconstruction of the ionizing tracks.

The task of reconstructing the tracks based on the signals from the pad plane is currently divided up into two parts, the first is that of determining the centroid of the energy deposited in the TPC and the amount of energy deposition, and the second is that of connecting the centroids ("hits") to form tracks. This study was devoted to testing and improving the second part.

\subsection{Tracking performance}

The input data for the nuclear collisions were taken from a FREESCO simulation of 1,000 1.0 A GeV Au + Au collisions and, for the later simulations, a FREESCo simulation of 1,000 1.2 A GeV Au + Au collisions was used, since a data set at that energy was aequired while running at the Bevatron. It is felt that the track finding will be insensitive to this small change in the beam energy and, in any case, the second set of data is more representative of the actual data. The FREESCO simulation was chosen since it is fast and reproduces most features of nuclear collisions. A detailed simulation of the nuclear collision is unnecessary for studying the track-finding software. The output from the FREESCO simulation is a list of the particle types and their momenta.

The GEANT package (from CERN) was used to simulate the physical processes of the 
particles passing through matter. A detailed simulation of the TPC's geometry was added, since this can be of general use to the EOS collaboration for other simulations of other aspects of the experiment. The detailed geometry also allowed locating and removing a $2.3 \mathrm{~cm}$ survey error in the placement of the TPC. The output from GEANT is the amount of energy deposition and its location in the TPC. A slow simulator exists to simulate the drift of the electrons, their amplification, and collection, but it was found that the simulator was too slow to allow statistically significant numbers of events to be simulated. A fast simulator was written with empirical simulations of the resolution effects and the hit merging due to overlapping ionization depositions. In addition to speed, other benefits of this approach are that the resolution is known exactly, and that any of the effects can be enabled or disabled as desired. In the data reported here the effects of resolution and merging are always enabled.

The performance of the track finder averaged over all particles present simulation (predominately protons), is shown in Table 1 . The first group of rows shows the performance of the track finders in using the hits to find the GEANT tracks. The first row is the number of tracks that contribute 20 or more hits in the TPC. There is a variation in this number as the geometry of the TPC was improved, and as the simulation of random processes varied from run to run. The second row is the fraction of the tracks which are not used. Here "used" is defined as this GEANT track contributes the majority of the hits to one of the track finder's tracks. The unused tracks' hits were not used by the track finder, or the hits were split up and assigned to other tracks. The third row is the fraction of the tracks that were used once. These tracks have the potential to be found correctly. The fourth row is the fraction of tracks that were used twice. A track that is used twice contributes the majority of the hits to two of the track finder's tracks - the track was split by the taack finder. The last row in this group is the fraction of tracks that were used three or more times. The first step in verifying good performance of the track finder is to verify that most of the tracks get used once and only once. Examining the entries shows that the earliest version of the track finder tended to split tracks, which was reduced in the later track finders.

Split tracks are particularly damaging to the proposed Hanbury-Brown-Twiss analysis. The two halves of the split track are found with similar (in principle, identical) momenta 


\section{TRACK FINDING STUDIES IN THE EOS TIME PROJECTION CHAMBER}

and, therefore, mask the HBT signal. Further, in this experiment the resolution of the TPC is about a factor of five times better than the HBT signal. Couple this with the strong length dependence of the resolution $\left(\frac{\delta P}{P} \approx l^{-5 / 2}\right)$, and one can see that the momentum difference spectrum of the split tracks has a similar width to the expected HBT signal (assuming that the track is split in half, the resolution for the parts is worse by a factor of $\left(\frac{1}{2}\right)^{-5 / 2} \approx 5.6$ ), and it is impossible to make cuts in the momentum difference to remove split tracks and leave an $\mathrm{HBT}$ signal.

Since the HBT signal rate (e.g., the rate of pion pairs with low momentum difference) is a small fraction of the single pion rate, even a small rate of track splitting will dominate the HBT signal. For this reason considerable effort has been expended on eliminating split tracks based on the hit information. Considering the fraction of input tracks used once as a measure of the number of split tracks, one can see that there was a large improvement between the first and second track finders, a slight decrease in performance between the second and the third, as the authors concentrated on improving the over-all efficiency, and a final improvement in the last track finder, which has the lowest rate of all for splitting tracks.

The second group of rows shows the performance of the track finders in not confusing the hits from different tracks. The first row is the number of tracks that were reconstructed to be 20 or more hits long. The second row is the fraction of those tracks that had all the hits assigned to this track from the same GEANT track. The third row is the fraction of the reconstructed tracks that contain hits from two GEANT tracks, and so on. In these rows, if a track is split, and the both halves are found, both halves will be considered separately and this will inflate the number of found tracks, possibly giving apparent efficiencies greater than one.

- Before one concludes that the first track finder is the best, one should notice that the rate of finding tracks is very much lower than the later track finders, and also that a single hit from another track is enough to make a track considered to have hits from other tracks. The ownership of merged hits is assigned to the input GEANT track that contributes the largest fraction of the energy deposition, and it is very likely that the merged hit is near both of the tracks, so it is possible that a merged hit can be assigned to the "wrong" track without introducing a significant error in the position of the track. 
1 TRACK FINDING STUDIES IN THE EOS TIME PROJECTION CHAMBER

\begin{tabular}{|c|c|c|c|c|}
\hline Version of track finder & 8-Jun-93 & 18-Jan-94 & 11-Jul-94 & 9-Nov-94 \\
\hline $\begin{array}{l}\text { GEANT tracks with } \geq 20 \text { hits in } \\
\text { the TPC. } \\
\text { Of these tracks: }\end{array}$ & 87,180 & 90,503 & 93,410 & 93,122 \\
\hline Not used by the track finder & $9.9 \%$ & $3.5 \%$ & $1.6 \%$ & $1.6 \%$ \\
\hline Used once by the track finder & $71.2 \%$ & $95.3 \%$ & $93.3 \%$ & $97.0 \%$ \\
\hline Used twice by the track finder & $18.0 \%$ & $1.0 \%$ & $4.6 \%$ & $1.4 \%$ \\
\hline Used $\geq 3$ times by the track finder & $0.8 \%$ & $<0.1 \%$ & $0.4 \%$ & $<0.1 \%$ \\
\hline $\begin{array}{l}\text { Tracks with } \geq 20 \text { hits in the } \\
\text { reconstructed track } \\
\text { Of these tracks: }\end{array}$ & 67,317 & 86,695 & 91,886 & 90,212 \\
\hline All hits come from one GEANT track & $96.6 \%$ & $86.9 \%$ & $71.8 \%$ & $73.9 \%$ \\
\hline Hits come from two GEANT tracks & $3.2 \%$ & $11.6 \%$ & $20.0 \%$ & $18.8 \%$ \\
\hline Hits come from $\geq 3$ GEANT tracks & $0.3 \%$ & $1.5 \%$ & $8.2 \%$ & $7.3 \%$ \\
\hline $\begin{array}{l}\text { Tracks with } \geq 20 \text { hits in the } \\
\text { reconstructed track and } \\
\geq 90 \% \text { of the hits from the } \\
\text { same GEANT track. }\end{array}$ & 65,889 & 82,496 & 82,718 & 82,515 \\
\hline As a fraction of the input tracks & $75.6 \%$ & $91.2 \%$ & $88.6 \%$ & $88.6 \%$ \\
\hline $\begin{array}{l}\text { Tracks with } \geq 20 \text { hits in the } \\
\text { reconstructed track and } \frac{\left|\delta P_{x}\right|}{\left|P_{\text {sotal }}\right|}<0.05 \\
\text { (similarly for } y \text { and } z \text { ). }\end{array}$ & $\begin{array}{c}55,476 \\
!\end{array}$ & 79,958 & 78,991 & 80,678 \\
\hline As a fraction of the input tracks & $63.6 \%$ & $88.3 \%$ & $84.6 \%$ & $86.6 \%$ \\
\hline As a fraction of the output tracks & $82.4 \%$ & $92.2 \%$ & $86.0 \%$ & $89.4 \%$ \\
\hline
\end{tabular}

Table 1: Track finding using 1.0 A GeV Au + Au FREESCO data as input to GEANT and the EOSGX fast simulation (first and second columns), and using 1.2 A GeV Au + $\mathrm{Au}$ FREESCO data as input (third and fourth columns). 


\section{TRACK FINDING STUDIES IN THE EOS TIME PROJECTION CHAMBER}

It is therefore necessary to relax this condition somewhat to be more realistic and allow a few hits from other tracks.

The next group of numbers relaxes the number of stray hits a bit, here the requirement is that $90 \%$ of the hits come from a single GEANT track, while keeping the requirement of 20 or more hits being present in the track. This is intended to be a figure of merit for the track finder recognizing a track and assigning the hits correctly to the track. Between the first and second track finders, the algorithm for finding tracks was changed; and the efficiency for finding tracks improved. Between the second and the third track finders, the number of tracks found is slightly reduced, reflecting the looser cuts used in an attempt to increase the number of tracks found, which increases the number of stray hits in the tracks. A comparison of the third and the last track finder shows that the reduction in the rate for finding split tracks was not made by further increasing the rate of including stray hits.

The last group of numbers requires that there are 20 or more hits in the reconstructed track, and the error in the components of the momentum obeys $\frac{\left|\delta P_{x}\right|}{\left|P_{\text {total }}\right|}<0.05$, $\frac{\left|\delta P_{y}\right|}{\left|P_{\text {total }}\right|}<0.05$, and $\frac{\left|\delta P_{z}\right|}{\left|P_{\text {total }}\right|}<0.05$, where $\delta P_{x}$ is the $x$ component of difference between the reconstructed and the input momenta (similarly for $\delta P_{y}$ and $\delta P_{z}$ ), and $P_{\text {total }}$ is the momentum input. Note that the error in each of the components is allowed to be $5 \%$ to the magnitude of the total momentum, not $5 \%$ of that component. This was done to allow for tracks that have $P_{x}$ or $P_{y}$ nearly zero. This was intended to be an overall figure of merit for the track finders since this is the number of tracks that would be output in the case of finding a cut that exactly correlates with a good momentum reconstruction. In reality, since the cuts only partially correlate with a good reconstruction, the number of tracks output would be less, or the momentum error would be larger. Between the first and the second track finders the major part of the increase in the number of found tracks with good momentum resolution is due to the increase in the number of tracks found. The later track finders show a slight decrease in the number of well found tracks as a fraction of the number of input tracks, which is believed to be due to the looser cuts employed.

The performance of the track finders for the negative pions in the simulated FREESCO events is shown in Table 2. The setup is similar to the first. However, only those tracks that match up to what was input as a negative pion are considered. Note that the 


\section{TRACK FINDING STUDIES IN THE EOS TIME PROJECTION CHAMBER}

multiplicity of found pions is only about four per event, so to simulate a statistically significant sample, a large data set is necessary, and a fast simulator is essential. The first row is the number of pions in the 1,000 FREESCO events with 20 or more simulated hits in the TPC. The second row is the number of tracks that were reconstructed with more than 20 hits, and the majority of the hits came from a negative pion track. Note that if tracks are split it is possible to find more pion tracks than were input (as is the case in the second and third columns). The third row requires that $90 \%$ of the hits in the reconstructed track come from the same GEANT track. This shows how well the track finder recognizes the tracks and assigns the hits to the track. The last row requires that there are 20 or more hits in the reconstructed track, and $\frac{\left|\delta P_{x}\right|}{\left|P_{\text {total }}\right|}<0.05, \frac{\left|\delta P_{y}\right|}{\left|P_{\text {total }}\right|}<0.05$, and $\frac{\left|\delta P_{z}\right|}{\left|P_{\text {total }}\right|}<0.05$. This is the number of tracks that could be output assuming that cuts could be found to remove only the poorly reconstructed tracks, and is intended to be a figure of merit for the track finders as applied to finding pion tracks. The different versions of the track finder show steady improvement. Note that split tracks may inflate the number of tracks found, and so the slight loss from the third to the last track finder is expected as the splitting of tracks is reduced. The implied rate for pairs of pions improved from $39 \%$ (for a track finder not in the table) to $68 \%$, to a final value of $79 \%$, thus the amount of data available has been doubled, and biases in the data have been reduced. By using a cut on the fraction of pad rows firing of the total number of pad rows crossed, the remaining split tracks can be completely eliminated in the Monte Carlo simulation with very small losses in the non-split tracks.

\subsection{Track finding code optimizations}

A secondary effort during the track finding studies was an attempt to increase the speed of the codes. This was motivated by the observation that, with the codes and processors available in the beginning of 1994, the time required to process all the data was greater than the probable lifetime of the experiment. Thus it is likely that the fraction of the data acquired in the Bevalac running that will be analyzed will be limited by the real time required to process the data, and the entire data set will not be analyzed.

The results of the timing tests are given in Table 3 . The time required to process an event has been reduced by a factor of two. This decrease in processing time has been 
1 TRACK FINDING STUDIES IN THE EOS TIME PROJECTION CHAMBER

\begin{tabular}{|c|c|c|c|c|}
\hline Version of track finder & 8-Jun-93 & 18-Jan-94 & 11-Jul-94 & $9-$ Nov-94 \\
\hline $\begin{array}{l}\text { GEANT } \pi^{-} \text {tracks with } \geq 20 \text { hits } \\
\text { in the TPC. }\end{array}$ & 3767 & 4004 & 5004 & 4934 \\
\hline $\begin{array}{l}\text { GEANT } \pi^{-} \text {tracks with } \geq 20 \text { hits } \\
\text { in the reconstructed track }\end{array}$ & $96.2 \%$ & $100.5 \%$ & $102.4 \%$ & $97.2 \%$ \\
\hline $\begin{array}{l}\text { GEANT } \pi^{-} \text {tracks with } \geq 20 \text { hits } \\
\text { in the reconstructed track and } 90 \% \\
\text { of the hits are from one GEANT track }\end{array}$ & $93.9 \%$ & $96.7 \%$ & $94.9 \%$ & $92.6 \%$ \\
\hline $\begin{array}{l}\text { GEANT } \pi^{-} \text {tracks with } \geq 20 \text { hits } \\
\text { in the reconstructed track and } \\
\frac{\left|\delta P_{z}\right|}{\left|P_{\text {total }}\right|}<0.05 \text { (similarly for } y \text { and } z \text { ) }\end{array}$ & $82.6 \%$ & $91.7 \%$ & $87.9 \%$ & $88.8 \%$ \\
\hline
\end{tabular}

Table 2: Track finding for the negative pions using 1.0 A GeV Au + Au FREESCO data as input to GEANT and the EOSGX fast simulation (first two columns), and using $1.2 \mathrm{~A} \mathrm{GeV}$ $\mathrm{Au}+\mathrm{Au}$ FREESCO data as input (last two columns).

accomplished without changing the algorithms involved, and so the performance of the tracker has not been compromised. The timing tests were done for the FREESCO data set of $1.2 \mathrm{~A} \mathrm{GeV} \mathrm{Au}+\mathrm{Au}$, since this data set is thought to be the most time consuming to analyze. It is believed that for multiplicities larger than perhaps 10 or so, the increase in speed will hold for the other data sets. Note that the production data processing is being done on SUN microsystems machines, and there are additional elements to the data analysis not being used in the Monte Carlo simulations, so that the time per event is different for the analysis of the real data. However, the time required to analyse data events is dominated by the track finding time, so it is believed that the relative speeds of the codes are close to that in the table.

\begin{tabular}{|lrrrr|}
\hline Version of track finder & 6-Sep-94 & 27-Oct-94 & 3-Nov-94 & 9-Nov-94 \\
\hline Time to track find an event (minutes) & 2.59 & 1.77 & 1.15 & 1.13 \\
\hline
\end{tabular}

Table 3: Track finding times of a VAX station $4000 \mathrm{M} 90$, for a FREESCo simulation of $2001.2 \mathrm{~A} \mathrm{GeV} \mathrm{Au}+\mathrm{Au}$ events, using the EOSGX fast simulator. 


\section{A preliminary Hanbury-Brown-Twiss analysis of the 1.2 $\mathrm{A} \mathrm{GeV} \mathrm{Au} \mathrm{+} \mathrm{Au} \mathrm{data}$}

The larger part of this analysis was done using the track finder of August 1993. The track finder has since been improved, as mentioned in Section 1.1, and a "bug" that produced tracks that exited the TPC at separations smaller than the hit merging distance was eliminated. The second effect was producing tracks with small relative momenta in the proton-proton as well as the pion-pion correlation analysis. Note that for the proton-proton analysis, Fermi-Dirac statistics apply, and the theoretical correlation function approaches 0.0 as the relative momentum decreases and it is thus unlikely that the effect was due to unusual physics in the nuclear collisions.

A large fraction of the $1.2 \mathrm{~A} \mathrm{GeV} \mathrm{Au}+\mathrm{Au}$ data set was analyzed. The less central of the two central trigger data sets was used since a data sample existed at the start of the data processing (which was made for another anailysis). In total 119,774 triggers were analyzed. About 35,914 events were found in the data set after cuts requiring the pions originate from the main interaction vertex, that the vertex was located near the target, and that two or more pions be present in the event.

In an attempt to eliminate the peak seen at low momentum differences seen during Monte Carlo tracking studies, a cut was made requiring that the transverse (to the beam) momentum difference be greater than $20 \mathrm{MeV} / \mathrm{c}$. A further cut was imposed requiring that the relative momentum difference be less than $300 \mathrm{MeV} / \mathrm{c}$ in any component, since the correlation function is essentially flat at this point and no further information is gained by keeping such pion pairs. About $1 / 2$ of the pion pairs pass these cuts. The cut at low momentum separations is bothersome since it is removing precisely the pion pairs that one wishes to fit.

The parameters from this fit are given in Table 4. The $\lambda$ parameter is a bit lower than typical for such fits, usually it is around 0.7 to 1.0. The source parameters are also a bit smaller than usual. The $\tau$ parameter is poorly constrained. The confidence level calculated for this fit is $10 \%$. It is believed that this fit demonstrates that it is possible to fit a correlation function to the $1.2 \mathrm{~A} \mathrm{GeV} \mathrm{Au}+\mathrm{Au}$ data, but further work has to be undertaken to improve the performance of the track finder. 


\begin{tabular}{|lrr|}
\hline Date & Aug 1993 & Oct 1994 \\
$\lambda$ & $0.51 \pm 0.1$ & $0.4 \pm 0.2$ \\
$R_{\|}$ & $3.3 \pm 0.5$ & $0.8_{-0.8}^{+1.6}$ \\
$R_{\perp}$ & $3.0 \pm 0.5$ & $2.9 \pm 0.8$ \\
$\tau$ & $1.3_{-1.3}^{+1.5}$ & $6.7 \pm 2.5$ \\
$\chi^{2} / N D F$ & $177 / 157$ & \\
\hline
\end{tabular}

Table 4: Correlation function parameters for $1.2 \mathrm{~A} \mathrm{GeV} \mathrm{Au}+\mathrm{Au}$ collisions fitted to about 35,000 pion pairs for the Aug 1993 data, and about 3,000 pion pairs (the second represents $17 \%$ of the data set).

Some modifications were done in the summer of 1994, as reported in Section 1.1. This work was largely complete in October 1994, when a data set was generated to search for systematic effects. Competition for the CPU resources within the group forced the choice of analyzing the smallest data set possible. A subset of 22,959 triggers from the $1.2 \mathrm{AgeV}$ $A u+A u$ central trigger data was analyzed. About 3,000 pion pairs were fit, and the extracted parameters are given in Table 4. The parameters are plotted along with the data for some other Bevalac experiments in Figures 1, 2, and 3. The small data sample size analyzed in the preliminary fit (about $1 / 7$ of the data set) does not allow making any definitive statements about the pion source parameters.

The correlation function is fitted in $q_{\|}, q_{\perp}, q_{0}$-space and is three dimensional. However, to allow plots to be made the data is averaged over two of the three dimensions, the fitted function is similarly averaged, and these real plotted as a function of the remaining variable. The plots of the projections onto the three coordinate axes are given in Figures 4, 5, and 6. Again, the statistics are limited, but no significant systematic effects are seen.

- Since the spectrometer was not triggered on two pions, the data set can be viewed also as a single pion data set. An approximate invariant cross section as a function of the pion energy in the center of mass of the colliding nuclei is plotted in Figure 7. Note that the only correction applied for the spectrometer's acceptance is requiring that $P_{z}$ be greater than zero in the center of mass (e.g., the pion had to be forward-going). This cut was made to try to force the pion to have high enough momentum in the laboratory that the TPC's acceptance was approximately flat. A fit to a single component exponential was 


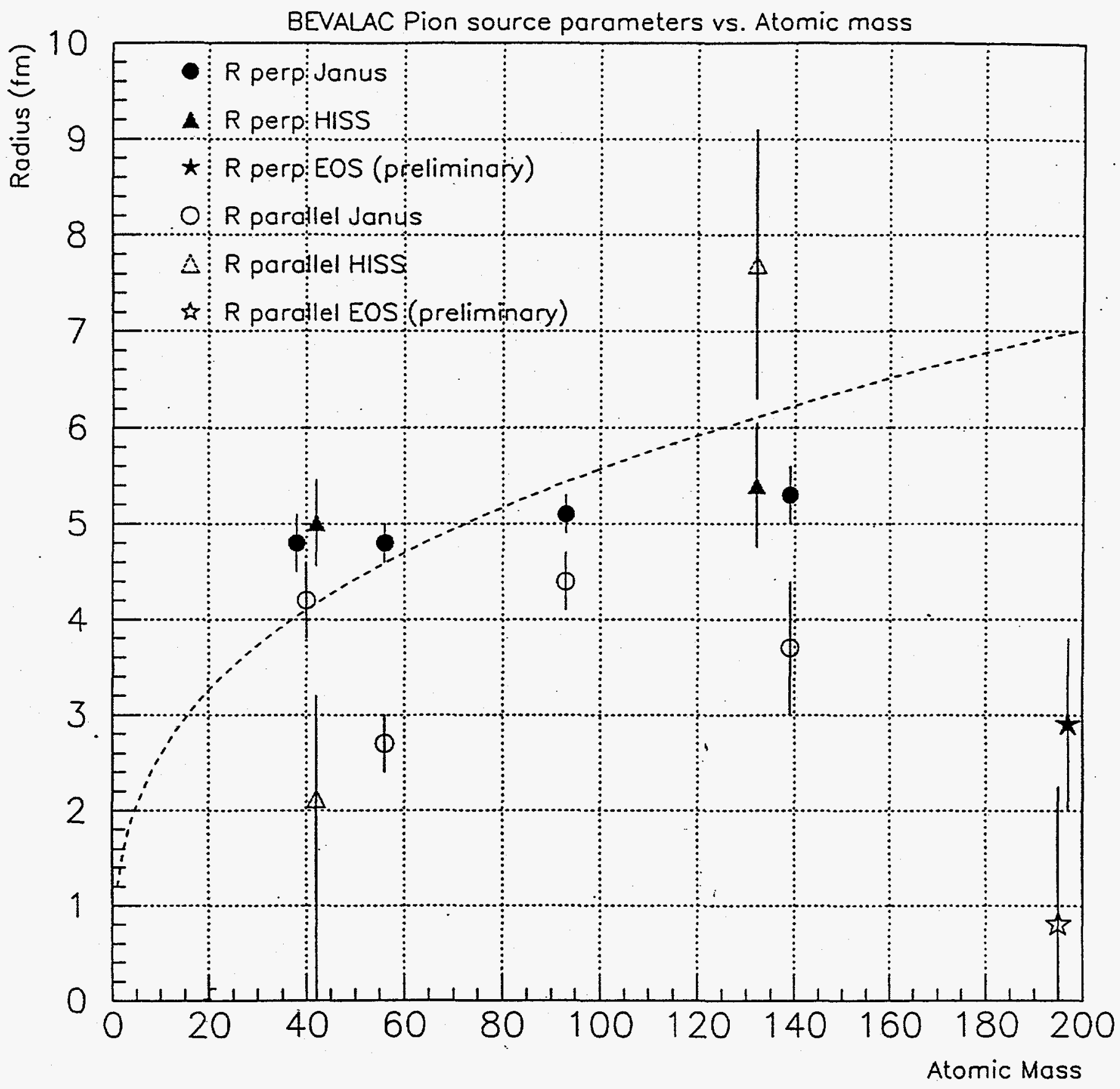

Figure 1: Radius parameters plotted as a function of atomic mass for some Bevalac experiments. 


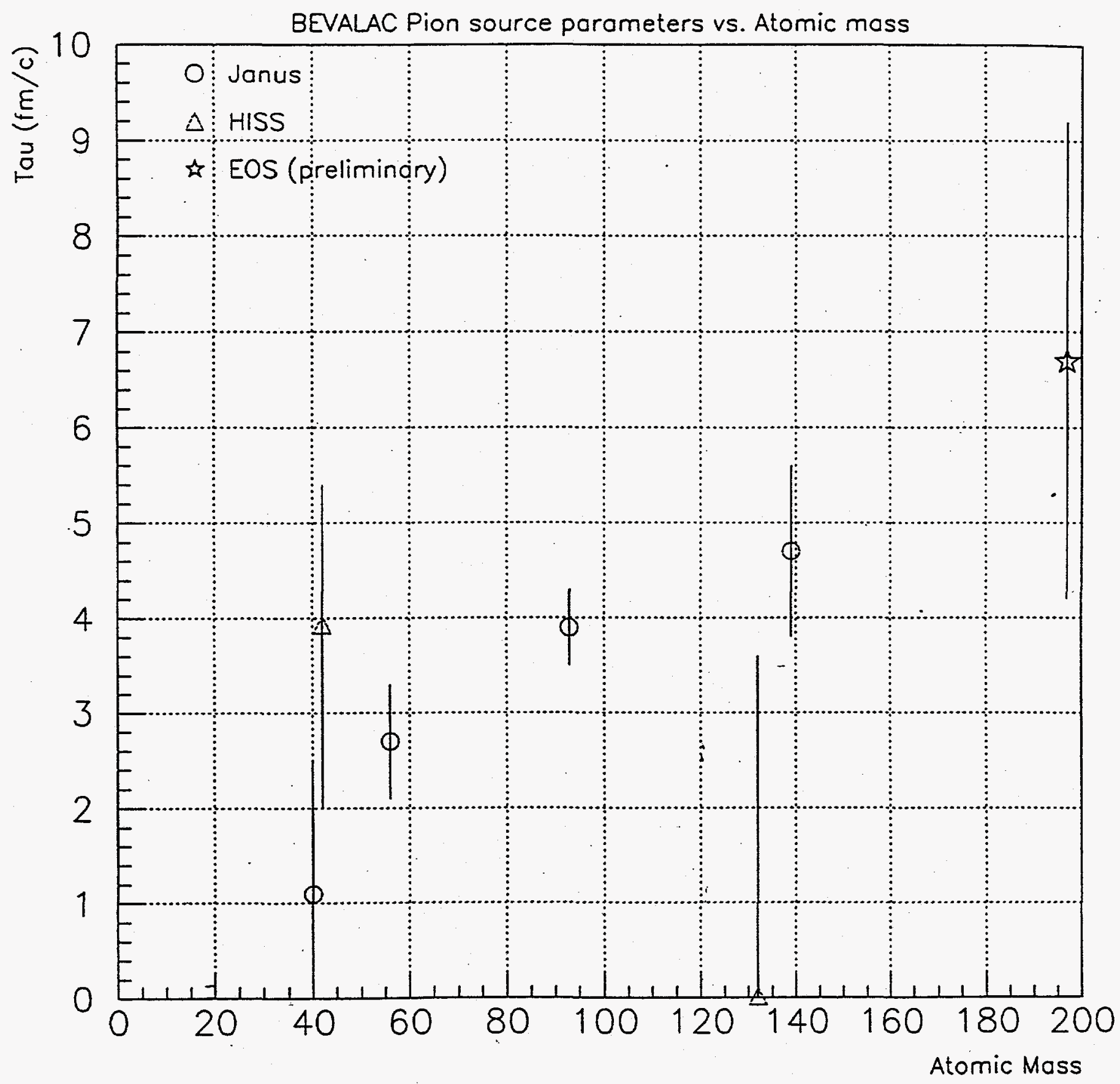

Figure 2: The $\tau$ parameter plotted as a function of atomic mass for some Bevalac experiments. 


\section{A PRELIMINARY HANBURY-BROWN-TWISS ANALYSIS}

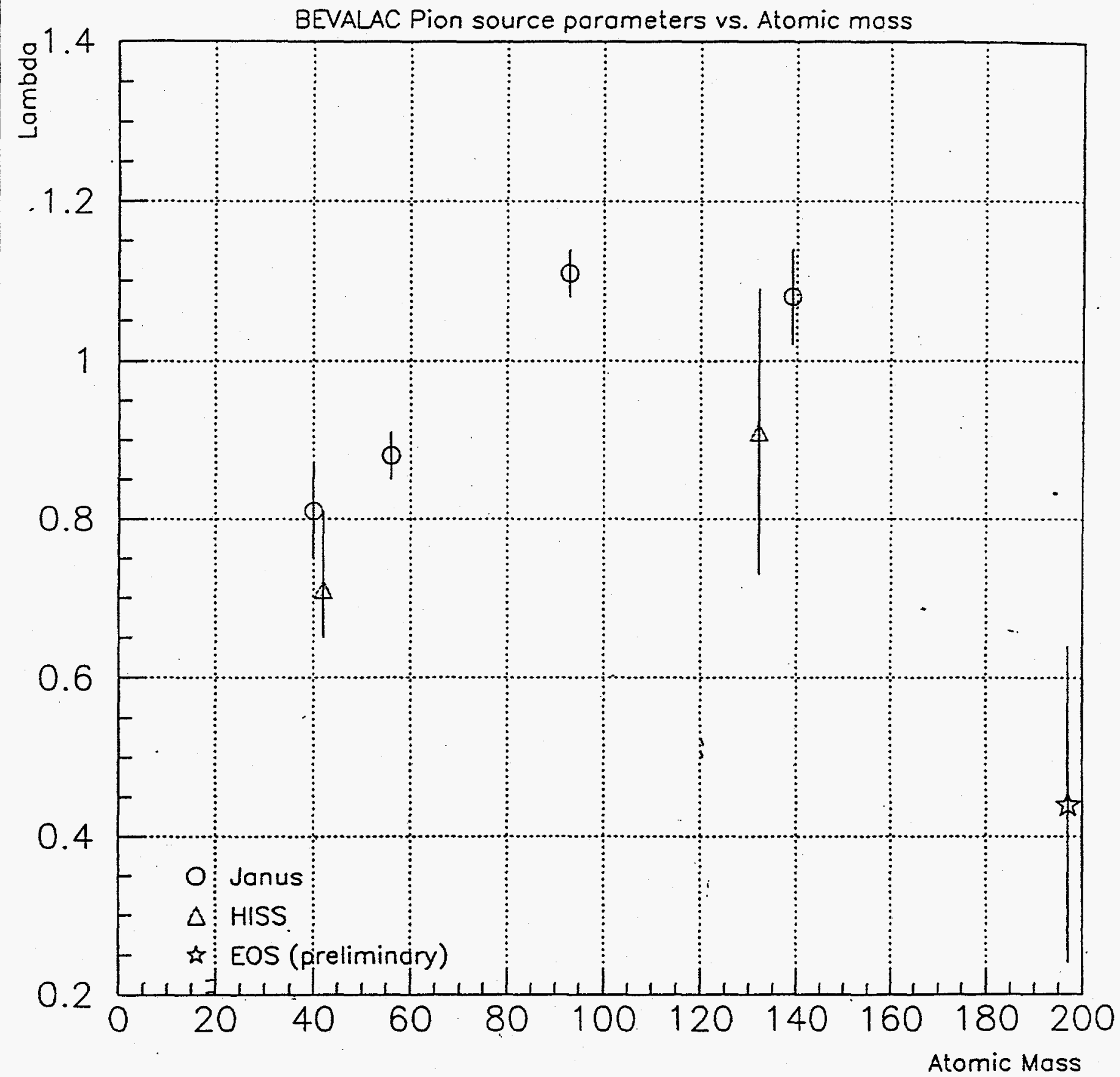

Figure 3: The $\lambda$ parameter plotted as a function of atomic mass for some Bevalac experiments. 


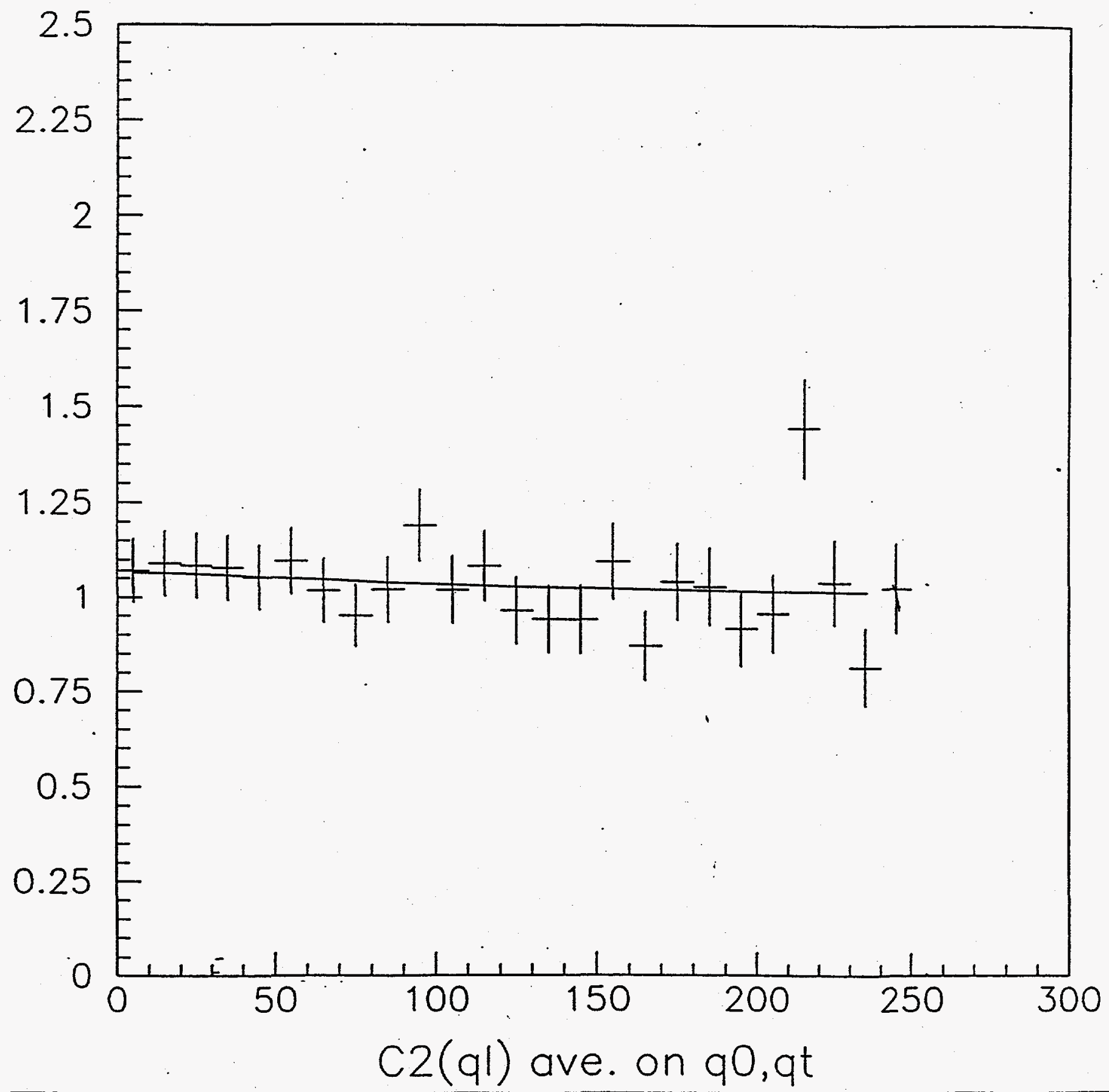

Figure 4: The projection of $C_{2}(q)$ on the $q_{\|}$axis. 


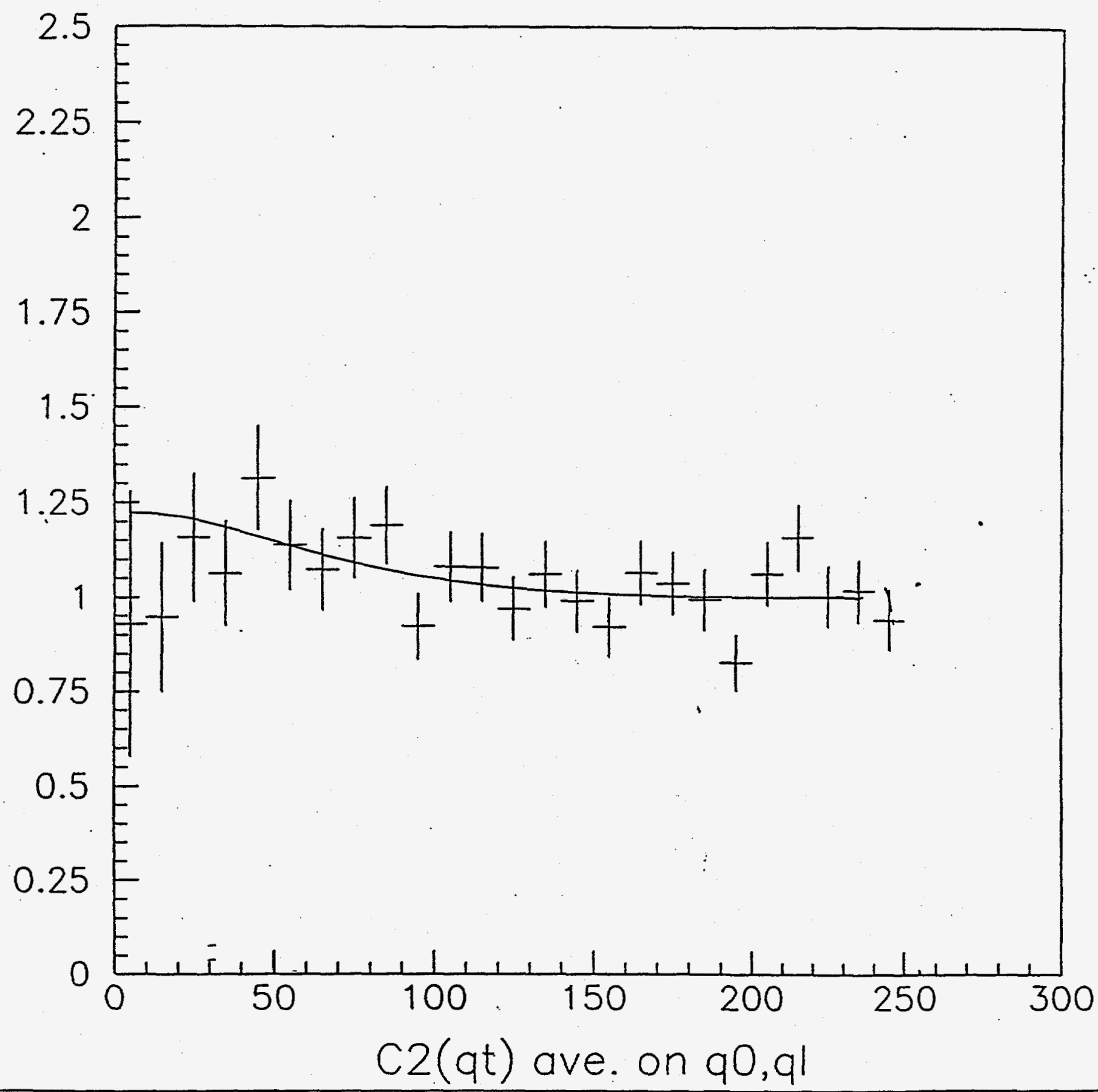

Figure 5: The projection of $C_{2}(q)$ on the $q_{\perp}$ axis. 


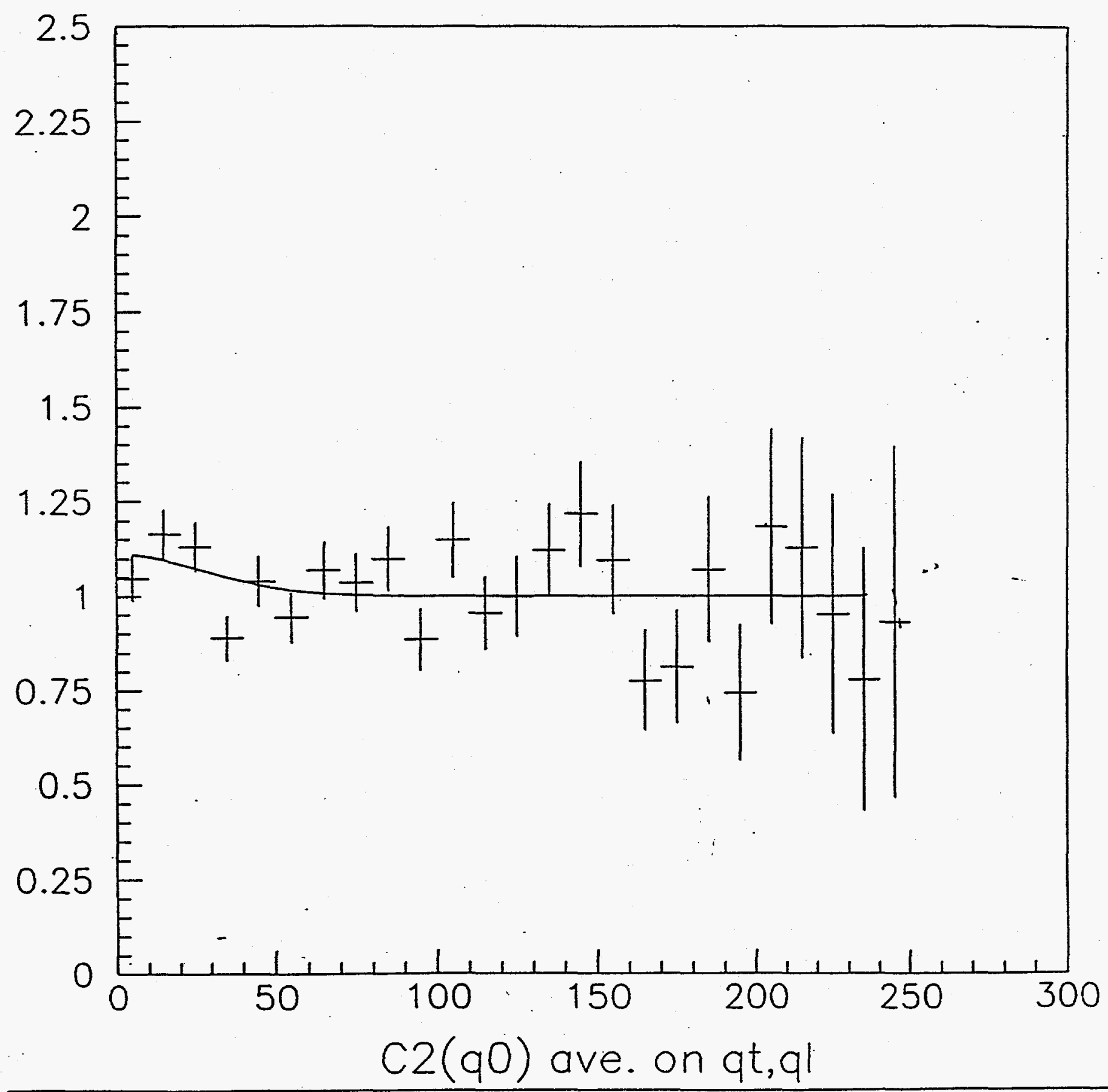

Figure 6: The projection of $C_{2}(q)$ on the $q_{\perp}$ axis. 
made and a slope parameter of $82.0 \mathrm{MeV}$ was extracted. The data in this plot represents a small fraction of the total data set. This analysis could also be applied to the smaller data sets (some of which are too small for the HBT analysis) taken at different beam energies to extract an excitation function.

The negative pion multiplicity is plotted in Figure 8. The average number of pions per event is 1.8. In FREESCO simulations with similar cuts the number of pions per event was 4.6 pions. This disagreement is under study since it has a large impact on the rate of pion pairs. 


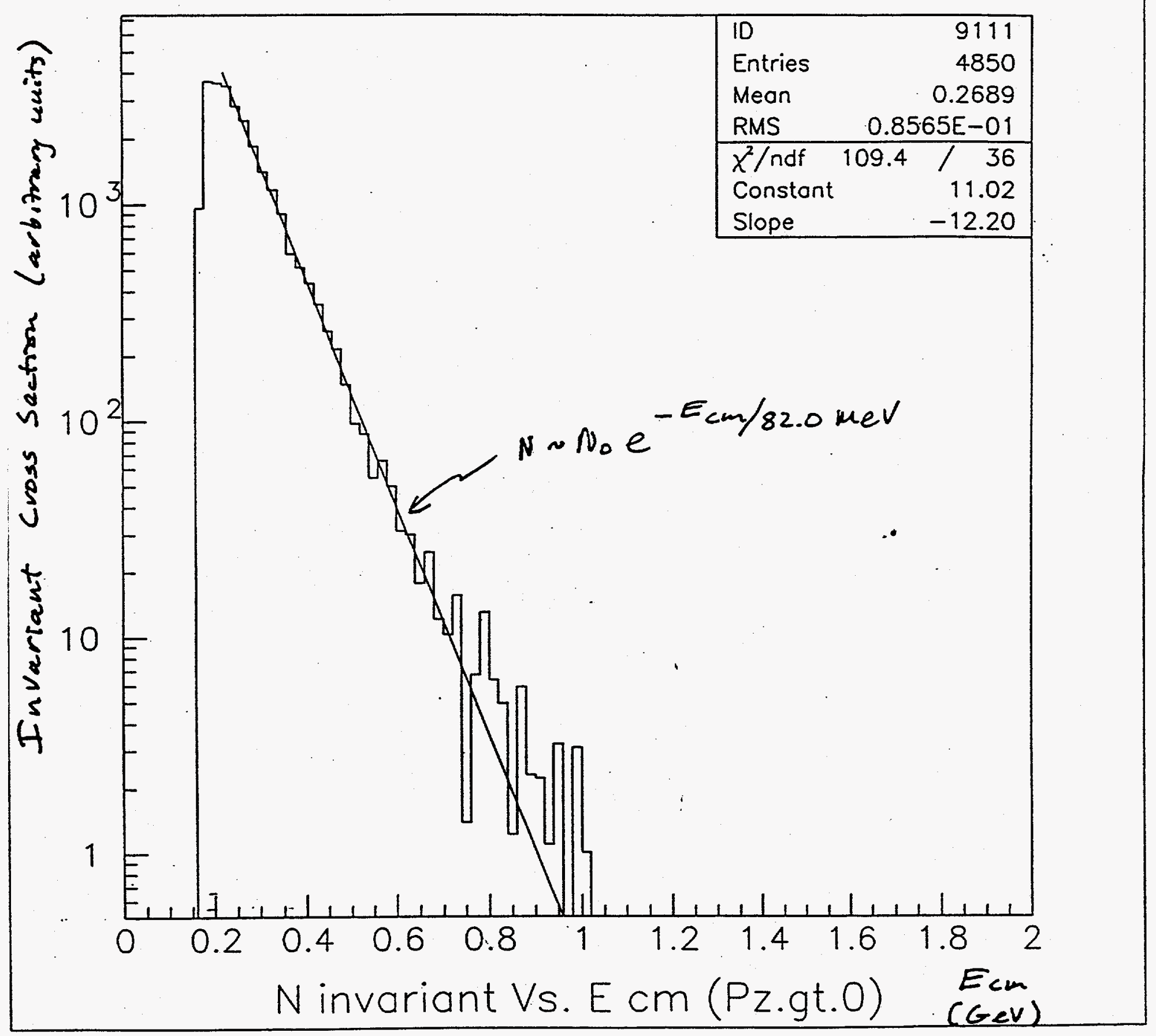

Figure 7: The invariant cross section (in arbitrary units) plotted as a function of the pion energy in the center of mass. 


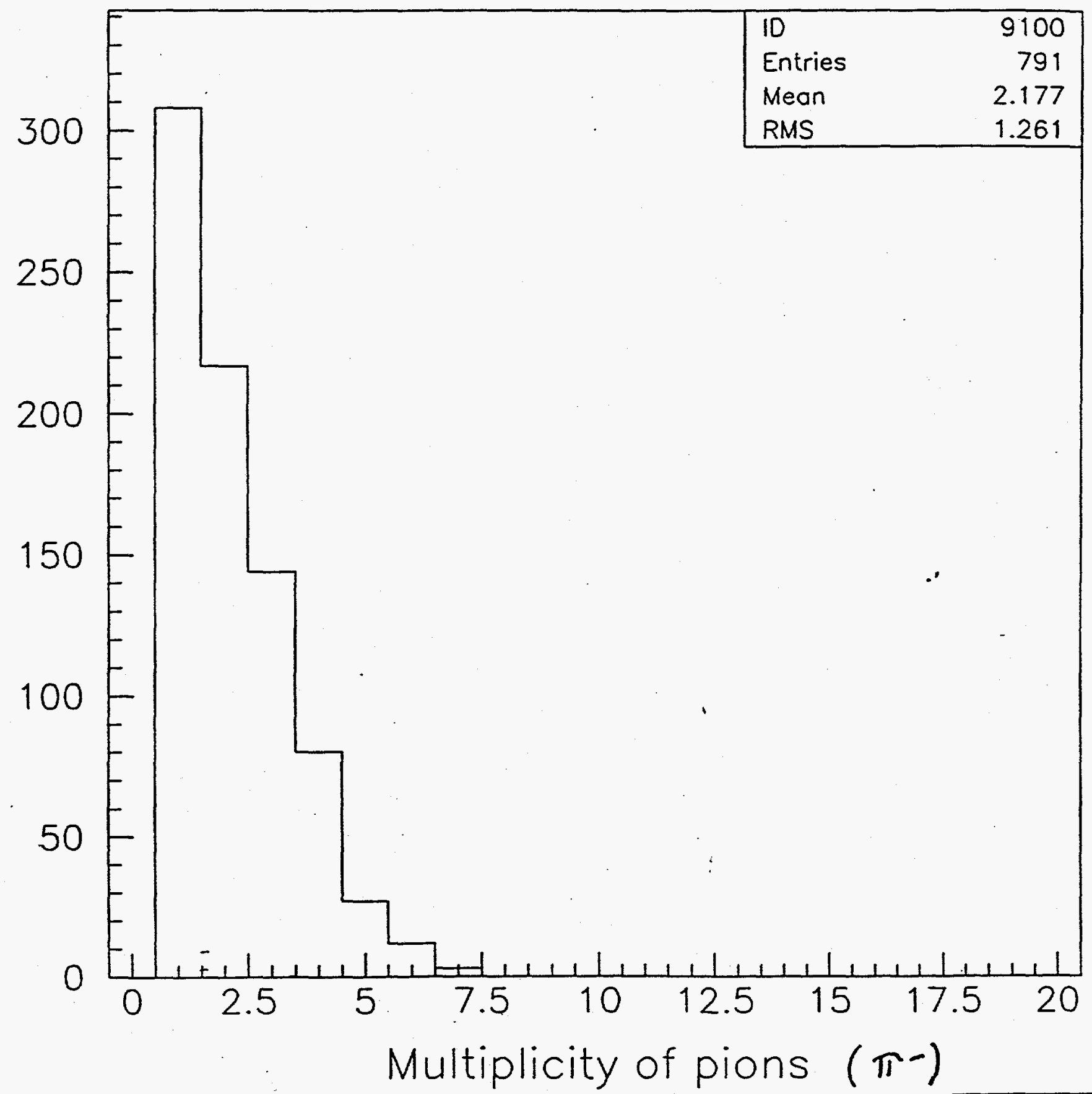

Figure 8: the $\pi^{-}$multiplicity for a subset of the 1.2 A GeV Au + Au data set. 


\section{SAMPLING CALORIMETER DEVELOPMENT AND TESTS}

An optical fiber readout scheme for sampling calorimetry was developed through the first stages at TAMU in 1987 before starting The Participant Calorimeter 1 project. The

original scheme outlined by Albrow, et al ${ }^{2}$ was extended to provide much higher, more reproducible response from plastic scintillator plates, into each of. which was bonded a single $1.5 \mathrm{~mm}$ diameter clad plastic optical fiber. The index of refraction of the bonding agent was matched to the fiber cladding. The green fiber used had the property of light absorption in the UV and blue, and reemission in the green after fairly efficient shifting. The light is then trapped and transmitted efficiently, with a transmission length of typically 15-20 meters. Most of the development time was spent on handling, grading and bonding fibers with little in the way for optimization of the method, since the photon response obtained was quite sufficient for a hadronic calorimeter. After some more recent development time on a DOE Generic Detector Project it is found that the response can be made as high as 20-30 photoelectrons per plate for mips through a $3 \mathrm{~mm}$ thick plastic scintillator and 5 pe response for $1 \mathrm{~mm}$ thick scintillator.

The superior response allows compensated hadronic calorimeters to use much thinner lead and scintillator plates, and thus should allow an increase in the energy resolution to that of uranium calorimeters. compact high resolution electromagnetic calorimeters can be constructed with the bonded fibers, which can use the good photoelectron statistics and uniform response. The disadvantages of the method include the labor intensiveness in bonding and stacking, and the dead regions caused by the fiber exit channels when one fiber per plate (or a few plates) is used. Also a large fiber bundle requires a large area readout device which is usually a phototube and always is expensive.

A solution to the readout problem was developed whereby the light from an entire calorimeter tower is concentrated into a single small optical fiber. Wavelength shifting fibers run perpendicular to the scintillator plane in a tile-type structure and a single $1.5 \mathrm{~mm}$ fiber can read out 75 (or more) plates as shown in Fig. 1 . In terms of an 
integrated solution the current scheme essentially eliminates dead regions, and allows the use of small active readout devices such as segmented anode phototubes as used here. The cost of the active readout device is then reduced by one order of magnitude per channel compared to conventional PMTs, which is most significant in an EM calorimeter. In the development project for a 100-tower test section constructed in our laboratory, a mip response of 5 photoelectrons per plate was obtained from $3 \mathrm{~mm}$ thick scintillator and a single $1.5 \mathrm{~mm}$ optical fiber readout. In the actual test section provision was made for up to 4-fold longitudinal segmentation which allows good particle identification over the 20-25 radiation lengths. An entire tower is read out by a single fiber, but additional light is collected from preshower and shower peak sections using fiber sections that are reflector coated to limit light collection to those desired longitudinal regions at the beginning or peak of the electron shower. The energy resolution is determined mainly by sampling fluctuations in the $2 \mathrm{~mm}$ thick lead absorber plates and was calculated to have a value of approximately $7 \% / S Q R T(E)$. Problems with uniformity of response in a similar early scheme ${ }^{3}$ have been solved by developing a "highly reflective" solution for the light collection as covered in the 1992 progress report. The light makes many passes (20-100) within the scintillator plate on average before collection, and thus the response becomes uniform transversely with regard to origin of the shower within a scintillator plate. Optimized scintillator, reflector and optical fiber combinations have reduced nonuniformities to the few percent level in bench tests. The good performance and the economy due to a small area readout device allow a high granularity necessary for measurement of electromagnetic energy in a high multiplicity such as at RHIC or the LHC, and to make electron shower size determinations for particle identification..

A series of resolution and response measurements were made recently on the calorimeter in a test beam at the CERN PS( line 7A) set up by the TAMU group during the summer of 1994. Pion, muon and electron beams were defined with high purity of the particle type through the use of two gas Cherenkov counters and time-of -flight scintillators. The beam momentum range was from $1-7 \mathrm{GeV} / \mathrm{c}$. In the first running of the calorimeter, four fibers per tower were run along the entire tower length, corresponding to 70 plates, totaling 21 radiation lengths. The apparent energy resolution sigms(E)/E for $3.0 \mathrm{GeV}$ electrons was approximately $8 \% / S Q R T(E)$, close to the predicted $7 \% / \mathrm{SQRT}(\mathrm{E})$, and not corrected for electron beam energy resolution. Energy calibrations of the towers were made initially with muons, and the calibration used a variational procedure to minimize the electon energy resolution over a cluster of towers. ( The tower size of $2.5 \times 2.5 \mathrm{~cm}$ never fully 
contained an elec

on shower,

as it sholud 1 ; for an effective $E M$ calorimeter.) An unexpected result came from the time resolution observed when the calorimeter timing was checked relative to the TOF counters in the beam line. For $3.0 \mathrm{GeV} / \mathrm{c}$ electrons, the measured resolution of the calorimeter was found to be 150ps, and this result held consistently over all towers and at $5 \mathrm{Gev} / \mathrm{c}$ as well as at $3 \mathrm{GeV} / \mathrm{c}$ electron energy. This time resolution is not surprising in retrospect, because of the large amount of light generated by the highly reflective scintillator plates and the efficient collection of the WLS optical fibers. Approximately $20 \mathrm{pe} / 3 \mathrm{~mm}$ plate/ mip results in a photoelectron count of 3000 pe $/ \mathrm{GeV}$ of electron shower energy deposited in the calorimeter. The solid possibility of a dual purpose device is suggested, to perform high resolution EM calorimetry and TOF array work. Time resolution may be improved by using available fibers that have a faster decay constant.

The calorimeter was reconfigured with a second set of optical fibers to make use of the (variable) longitudinal segmentation feature of the calorimeter design. Optical fibers were coated with a water soluble reflective paint in regions that light collection was not wanted. The fibers received a secondary clear coat to eliminate the possibility of reflective paint contamination in the scintillator holes upon stringing the fibers through the calorimeter plates. A longitudinal segmentation of 4,8 , and 9 radiation lengths was chosen, corresponding to preshower, shower peak and shower tail sections. A fourth fiber integrated the full depth of each calorimeter tower to anchor the test results. Normally the fourth fiber would be a clear fiber for injection of blue light for calorimeter gain stabilization. In the present tests green LEDs were used to monitor only the phototube gains. Good electron/pion separation was obtained and results are currently undergoing final analysis.

Table II provides a comparision of the present calorimeter test section with a similar design, in principle, completed several years ago 3,4 . The principal technical differences involve a highly reflective and efficient light collection scheme in the present design, and the use of computerized mills for the entire test section here. Additional benefits of the present design include the fast timing capability and a uniform lateral response across the calorimeter. 
TABLE II

Comparision of Calorimeter Parameters

$$
\text { Present Design (Loehr, et a }{ }^{4} \text { ) }
$$

number of fibers

4 (or 1$)$

16

per tower

Diameter of

$1.5 \mathrm{~mm}$

$1.5 \mathrm{~mm}$

fibers

Fiber material

polystyrene

Fiber clading

vinyl acetate

polystyrene

dopant

$200 \mathrm{mg} / 1 \mathrm{~K}-27$

PMMA

$400 \mathrm{mg} / 1 \mathrm{~K}-27$

Scintillator

$3 \mathrm{~mm}$ Pilot $\mathrm{F}$

$5 \mathrm{~mm} \operatorname{sCSN}-38$

Abs. plates

$2 \mathrm{~mm} \mathrm{~Pb}(\mathrm{~Pb}-\mathrm{Al}-\mathrm{Pb})$

$2 \mathrm{~mm} \mathrm{~Pb}$

Photoelecttrons

26 ( 6.6 single fiber)

1.7

per $\mathrm{MeV}$

Time resol

$150 \mathrm{ps}$

sigma/E

$7 \%$

$8.6 \%$

nonuniformity

$3 \%$

$35 \%$

of response (P/V)

\section{References}

1. J. Simon-Gillo, et al, NIM $\underline{A 525}$ 474:(1992) and NIM A317 474(1992).

2. M. Albrow, et al, Nucl. Phys. 1461 417C(1987).

3. H. Fessler, et al, NIM A240 284 (1985).

4. B. Loehr, et al, NIM A254 26(1987). 


\section{RIP CALURIMETER}

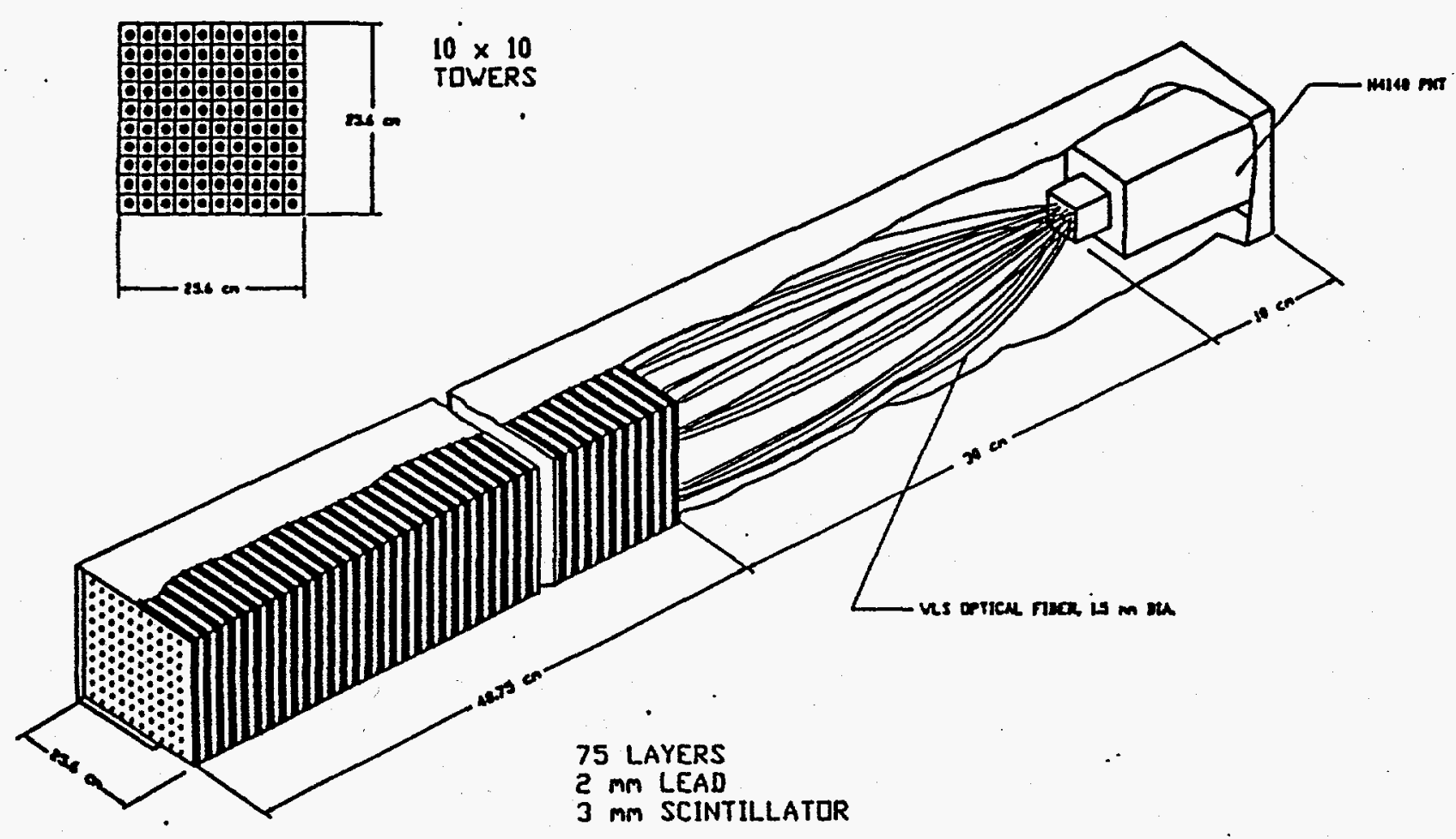

Figure 1 Schematic layout of the electromagnetic calorimeter tested during the summer of 1994 at the CERN PS. 


\section{EXPERIMENT NA44 AT CERN}

Collaborating Institutions: BNL, CERN, Columbia, Creighton, Hiroshima U., LANL, Lund, Tbiliisi state U., Tsukuba National Lab., Neils Bohr Inst., Ohio state, TAMU, Tokyo U., and Tsukuba U.

Fixed target operation at a beam energy of $200 \mathrm{~A} \mathrm{GeV}$ for ${ }^{32} \mathrm{~S}$ and $160 \mathrm{~A}$ $\mathrm{GeV}{ }^{208} \mathrm{~Pb}$ is the highest heavy-ion energy available before going to collider mode as planned at RHIC and the LHC. While well below the energy necessary for full transparency and the formation of a baryonfree high energy density region for the quark gluon plasma, the energy density should be significant, in the 2-3 GeV/ $\mathrm{fm}^{3}$ region and may be sufficient for the formation of a mixed-phase QGP. A realistic goal consists of investigating and understanding the features of heavy-ion reactions both at BNL and CERN, at energies lower than the future colliders in order to be able to understand those data in the future.

Identical particle interferometry can provide information about the dynamical evolution of heavy-ion reactions, specifically through the space-time extent of the emitting source. The time extent of particle emission can provide evidence for a phase transition as outlined by Bertsch and Brown ${ }^{1}$. The relatively high energy available at CERN allows interferometry measurements with several types of produced particles including pions and kaons, and to perform corelation measurements with protons and possibly antiprotons in the lead beam experiments. A comparision with model calculations such as RQMD leads to values of source sizes and shapes for different particles and momentum ranges, presumably corresponding to different stages of the reaction. An additional factor at CERN energies, is the large amount of resonance production and deexcitation that can confuse or dilute interferometry measurements, especially for pions. The resonance decay contribution is considered to be an important area of study in these experiments and so far has been handled through comparision with microscopic calculations such as $R Q M D$ and through $k_{t}$ cuts on pion energy. A second important type of measurement in the NA 44 experiments consists of identified single particle spectra, which are necessary in understanding the topology of the reactions. The shapes of the spectra and the rapidity distributions are a constant topic of discussion in the understanding of the dynamics of the reactions and even in the search for exotic effects.

NA44 is an example of a second generation experiment, which builds upon the results of the completed and analyzed survey experiments. 
The first phase of the apparatus was brought on line for $200 \mathrm{~A} \mathrm{GeV}$ sulfur beam periods at the SPS and for $450 \mathrm{GeV}$ proton beams. The general layout of the apparatus was designed for very heavy ion beams and is shown in Fig.1. Because of the increased multiplicity with lead beams and because of experience gained in the previous program, some new equipment had to be built for the lead beam sps capability that ran at the end of 1994. The general philosopy is one of attempting to measure a small slice of an event instead of a global measurement, and to make that measurement in an optimized manner. Thus the primary goal is to measure identified single paricle distributions and two particle distributions at or near mid-rapidity. The primary detector is a focusing spectrometer designed to accept multiple particles with a small momentum difference and uses a combination of three quadrupoles, three dipole magnets, and a series of TOF hodoscopes, MWPCs and Cherenkov counters to track and identify charged particles in the spectrometer. Thus pions, kaons, protons and deuterons both positively and negatively charged are identified and measured with good electron rejection. A typical data sample for pion pairs consists of 200,000 events. The primary impact parameter selector at the trigger level is furnished by a plastic scintillator that operates in a positive pulse height mode, i.e. as compared to a leading particle veto trigger. A silicon pad array is used off line to determine the charged-particle multiplicity over the rapidity range of $1.5-3.3$.

Progress on the analysis of singles data collected previously has, in the past year involved details in correcting inconsistancies in the measured spectra and the data have changed little from presentations a year ago $^{2}$.

The information content of identified singles spectra is somewhat debatable, but if one can define the amount of proton and antiproton production and differentiate from the incident baryons, in a known participant number collision, then constraints can be put on the stopping power, for instance, which is a crucial quantity at SPS energies. Defining the production part of the yields does, however, have significant problems. Unfortunately one only measures the total proton yield (and that averaged over a considerable impact parameter range in NA44) in S + $\mathrm{Pb}$ collisions, which results in protons at midrapidity form participants and secondary cascading, as well as from antiproton-proton production. Figure 2 shows a rapidity distribution from an RQMD calculation to illustrate this point. The NA44 measures the absolute value of $\mathrm{dN} / \mathrm{dy}$ near mid-rapidity and there is good agreement with Fig.2 for $S+P b$ and fair agreement for $S+S$. The antiproton yield (Fig.3) does not have the production contamination, 
but does suffer $f$ m annihilation, which low $;$ the yield by an amount that is extremely hard to calculate. One must know the baryon distribution to calculate the absorption, and this is much harder than at AGS energies with complete stopping. The scattering/annihilation of antiprotons off of partially stopped baryons, transferred target region baryons and off of the rich pion environment is in itself a complicated problem. It is not a mystery then, for these reasons that RQMD calculations must be modified to account for antibaryon and strange particle yields, which may not have to do with strings and ropes at all. The ${ }^{32} \mathrm{~S}+{ }^{32} \mathrm{~s}$ reaction suffers less from spectator matter complication, but is too light to be useful, especially for the rather weak impact parameter bias of b<3fm used in NA44, which provides only about 40 participant nucleons on average. It should not be surprising that the rapidity distribution for protons would show partial stopping, as it does for Si+ Al at lower AGs energies.

The spectral shapes of proton and antiproton spectra may contribute information on a type of "temperature" of the system and possible radial expansion or blast wave effects. Figure 4 shows the slopes from fitting exponentials through proton and antiproton $m_{t}$ spectra for proton-and sulfur-induced reactions. The $450 \mathrm{GeV}$ proton beam experiments used a weak trigger condition of a particle in the spectrometer and one or more particles in the $T_{0}$ trigger counter, thus the apparent teperatures, while not quite for minimum bias collisions, are nearly so and become more so with increasing target mass. Thus there is no target dependence for $p+N$ reactions since most events are from a thin skin region on the target surface. The semi-central triggered $32 \mathrm{~s}$ - induced reactions show enhanced multiple scattering through higher apparent temperatures compared the p-induced reactions. There is only a slight hint of a trend discernable for increased slope with system size in these proton emission data, disregarding p-induced data for reasons given above. In fact the antiproton data show the opposite effect when comparing $S+S$ with $S+P b$ reactions. The antiproton/proton slopes nearly agree for the lighter $s+s$ reaction, but a sizeable decrease in the slope ratio is seen for $S+P b$. It is tempting to simply use the fireball model, to explain the effect of a lower temperature in an asymmetric system, as seen for produced particles such as antiprotons.( The high apparent temperature for proton emission for $S+\mathrm{Pb}$ is attributed to multiple scattering in the target matter.) This must be tempered somewhat since there is incomplete stopping, certainly in $S+S$ and probably for $S+P b$. Absorption is expected to increase the antiproton apparent temperature because of higher annihilation cross sections at low antiproton energies, quite the opposite to the effect seen. Perhaps the cause of the reduced apparent temperature involves a baryon potential argument 
as put forth by Chi Ming Ko many years ago, since the dN/dy for baryons at mid-rapidity is quite high for $200 \mathrm{~A} \mathrm{GeV} \mathrm{S}+\mathrm{Pb}$, nearly as high as at AGS energies for $14 \mathrm{~A} \mathrm{GeV} \mathrm{Si}+\mathrm{Au}$. It should be noted that the NA44 spectrometer integrates protons and antiprotons from lambda and anti-lambda decay, and thus mixes strangeness and antibaryon phenomena.

\section{REFERENCES}

1.. G. Bertsch and G.E. Brown, Phys. Rev. C40 1830(1989).

2. B.V. Jacak, Proceedings of the NATO Advanced study Institute, Bodrun, Turkey, 1993. LA-UR-94-713

3.H. Sorge, et al, Ann. Phys. 192 266(1989). 

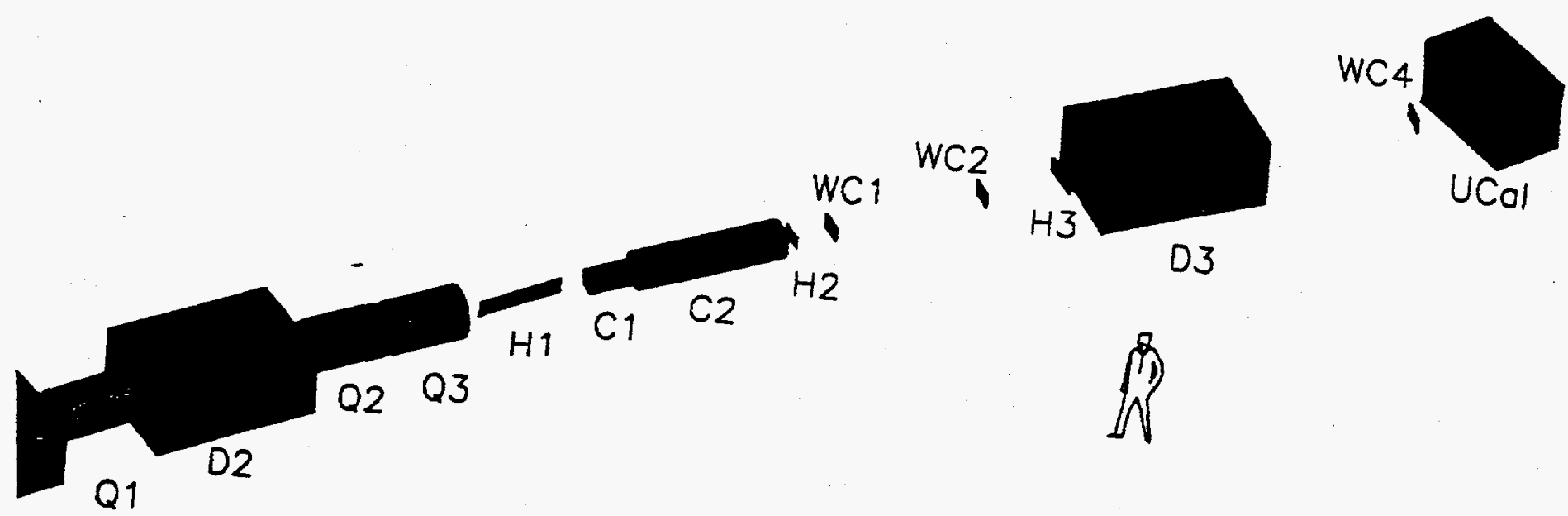

Figure 1 - NA 44 Focussing spectrometer $Q=$ superconducting quadrupole, $\mathrm{D}=$ dipole magnet, $\mathrm{H}=$ plastic scintillator hodoscope, $C=g a s$ Cherenkov counter, $W C=$ wire chamber, Ucal= uranium calorimeter

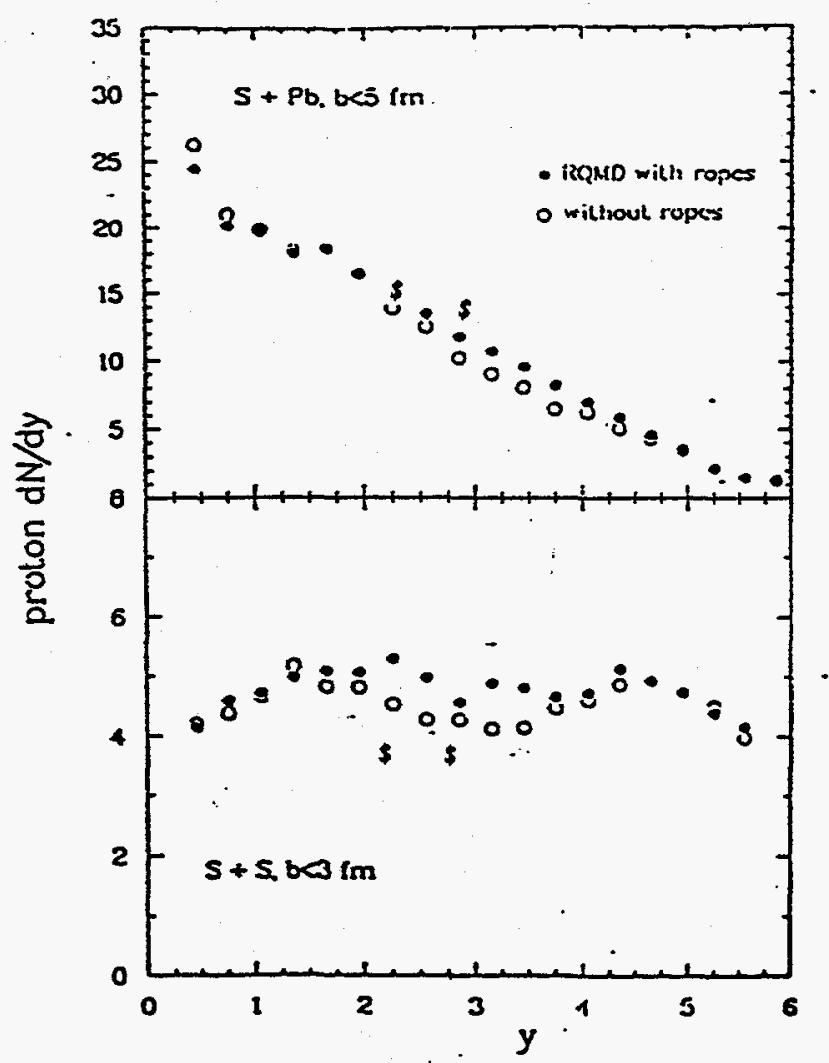

Figure 2 - Proton rapidity distributions calculated with $R_{Q M D}{ }^{3}$ for $200 \mathrm{~A}$ GeV $\mathrm{S}+\mathrm{Pb}$ and $\mathrm{S}+\mathrm{S}$. Experimental data from NA44 are shown as the $\$$ symbols. 


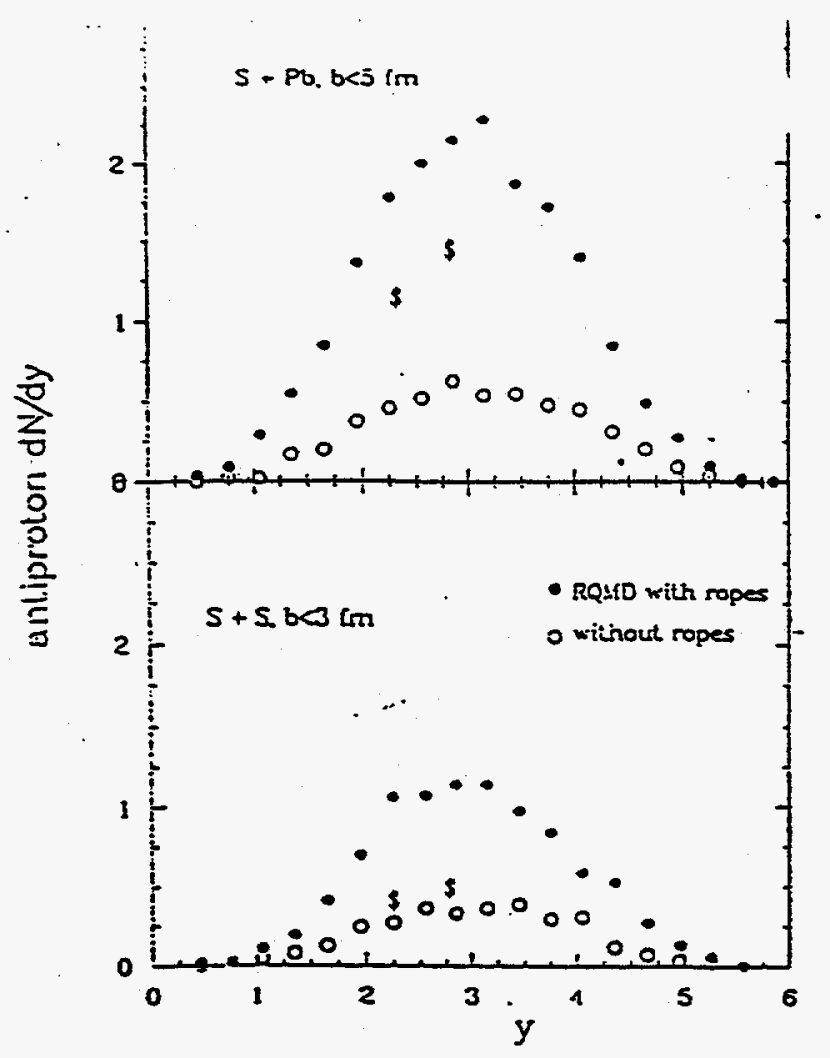

Figure 3 - Same as Fig.2 except for antiproton emission.

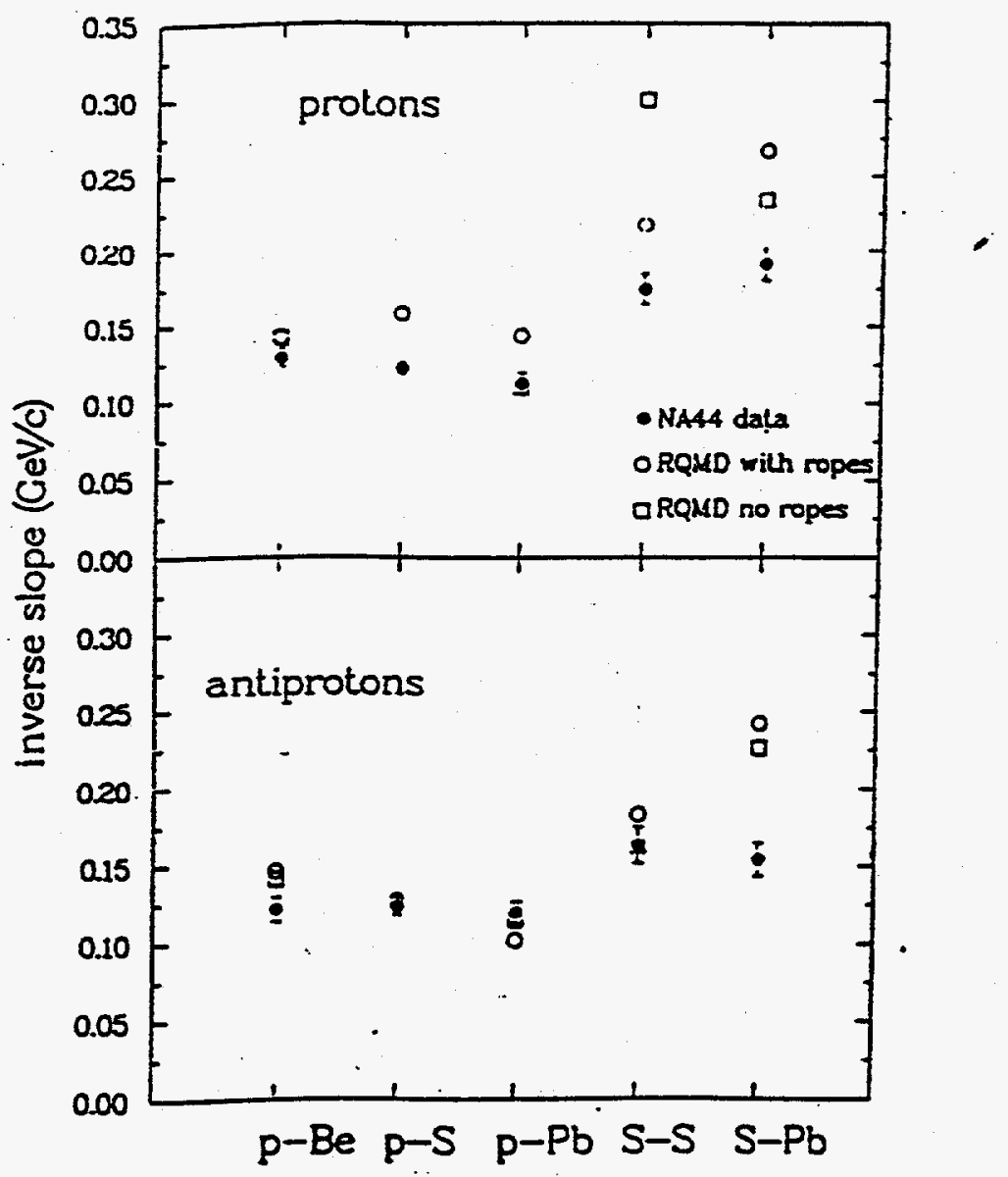

Figure 4 - The apparent tenperture extracted from $m_{t}$ spectra of protons and antiprotons near mid-rapidity for 450 proton and $200 \mathrm{~A} G \mathrm{GV}$ sulfur reaction on various targets. 
Midrapidity pion Ratios in $1.05 \mathrm{GeV} / \mathrm{nucleon}{ }^{40} \mathrm{Ca}+40^{\circ} \mathrm{Ca}$ Collisions, J.W. Harris, J. Miller, H.G. Pugh, P. Renteln, G. Roche, P.N. Kirk, G. Krebs, R. Brockmann and K.L. Wolf, Phys. Rev. C41 147 (1990).

Electromagnetic Dissociation of ${ }^{28} \mathrm{Si}$ at $\mathrm{E}_{1 \mathrm{ab}} / \mathrm{A}=14.6 \mathrm{GeV} / \mathrm{A}$ by Nucleon Emission, J. Barrette, P. Braun-Munzinger, W.E. Cleland, G. David, E. Duek, M. Fatyga, D. Fox, S.V. Green, J.R. Hall, R. Heifetz, T.K. Hemmick, N. Herrmann, R.W. Hogue, G. Ingold, K. Jayanada, D. Kraus, A. Legault, D. Lissauer, W.J. Llope, T. Ludlam, R.D. Majka, D. Mackowiecki, S.K. Mark, J.T. Mitchell, M. Muthuswamy, E. O'Brien, L.H. Olsen, V. Polychronakos, M. Rawool-Sullivan, F.S. Rontondo, J. Sandweiss, B. Shivakumar, J. Simon, U. Sonnarara, J. Stachel, J. Sunier, H. Takai, T.G. Throwe, H. VanHecke, L. Waters, W.J. Willis, K. Wolf, D. Wolfe and C. Woody, Phys. Rev.C41 1512 (1990).

Energy Flow and stopping in Relativistic Heavy-Ion Collisions at $\mathrm{E}_{\mathrm{lab}} / \mathrm{A}=14.6 \mathrm{GeV}$, J. Barrette, R. Bellwied, P. BraunMunzinger, W.E. Cleland, G. David, E. Duek, M. Fatyga, D. Fox, A. Gavron, S.V. Green, J. Hall, R. Heifetz, T.K. Hemmick, M. Herman, N. Herrmann, R.W. Hogue, G. Ingold, K. Jayanada, D. Kraus, A. Legault, D. Lissauer, W.J. Llope, T. Ludlam, R.D. Majka, D. Mackowiecki, S.K. Mark, J.T. Mitchell, M. Muthuswamy, E. O'Brien, L.H. Olsen, V. Polychronakos, M. Rawool-sullivan, F.S. Rontondo, J. Sandweiss, B. Shivakumar, J. Simon, U. Sonnarara, J. Stachel, J. Sunier, H. Takai, T.G. Throwe, H. VanHecke, L. Waters, W.J. Willis, K. Wolf, D. Wolfe and C.L. Woody, Phys. Rev.C41 1512 (1990).

Energy Flow and stopping in Relativistic Heavy-Ion Collisions at $\mathrm{E}_{1 \mathrm{ab}} / \mathrm{A}=14.6 \mathrm{GeV}$, J. Barrette, R. Bellwied, P. BraunMunzinger, W.E. Cleland, G. David, E. Duek, M. Fatyga, D. Fox, A. Gavron, S.V. Green, J. Hall, R. Heifetz, T.K. Hemmick, M. Herman, N. Hermann, R.W. Hogue, G. Ingold, K. Jayanada, D. Kraus, A. Legault, D. Lissauer, W.J. Llope, T. Ludlam, R.D. Majka, D. Mackowiecki, S.K. Mark, J.T. Mitchell, M. Muthuswamy, E. O'Brien, L.H. Olsen, V. Polychronakos, M. Rawool-sullivan, F.S. Rontondo, J. Sandweiss, B. Shivakumar, J. Simon, U. Sonnarara, J. 
Stachel, J. Sunier, H. Takai, T.G. Throwe, H. VanHecke, L. Waters, W.J. Willis, K. Wolf, D. Wolfe and C.L. Woody, Phys. Rev. Lett. 641219 (1990).

Search for strange Quark Matter in High-Energy Heavy-Ion Collisions, J. Barrette, R. Bellwied, P. Braun-Munzinger, W.E. Cleland, G. David, E. Duek, M. Fatyga, D. Fox, S.V. Green, J.R. Hall, R. Heifetz, T.K. Hemmick, N. Herrmann, R.W. Hogue, G. Ingold, K. Jayanada, D. Kraus, A. Legault, D. Lissauer, W.J. Llope, T. Ludlam, R.D. Majka, D. Mackowiecki, S.K. Mark, J.T. Mitchell, M. Muthuswamy, E. o'Brien, L. Olsen, V. Polychronakos, M. Rawool-Sullivan, F.S. Rontondo, J. Sandweiss, B. Shivakumar, J. Simon, A.J. Slaughter, U. Sonnarara, J. Stachel, J. Sullivan, J. Sunier, H. Takai, T. Throwe, H. Van Hecke, L. Waters, K.L. Wolf, D. Wolfe, and C. Woody, Phys. Lett. B252 530(1990).

Response of a Sampling Calorimeter to Low Energy Particles, M.W. Rawool-Sullivan, J. Shoemaker, J. Simon, J.P. Sullivan, K.I. Wolf, A. Archuleta, G. Barasch, P. Bennett, D. Bertini, J. Bossevain, K. Holtzscheiter, B. Jacak, W. Sondheim, J. Sunier, H. Van Hecke and B. Wolf, Nucl. Phys. A525 677c(1991).

Pion Correlations in Relativistic Heavy Ion collisions for Three symmetric systems, A.D. Chacon, J.A. Bistirlich, R.R. Bossingham, H. Bossey, H.R. Bowman, C.W. Clawson, K.M. Crowe, T.J. Humanic, M. Justice, P. Kammel, J.M. Kurck, S. Ljungfelt, C.A. Meyer, C. Petijean, J.O. Rasmussen, M.A. Stoyer, O. Hashimoto, Wm. C. Mcharris, J.P. Sullivan, K.L. Wolf and W.A. Zjac, Phys. Rev. C43 2670(1991).

Forward Baryon Emission and Charged Multiplicity in Si-N Collisions, J. Barrette, R. Bellwied, P. Braun-Munzinger, W.E. Cleland, G. David, M. Fatyga, D. Fox, S.V. Green, J.R. Hall, R. Heifetz, T. Hemmick, N. Herrmann, R. Hogue, G. Ingold, K. Jayanada, D. Kraus, D. Lissauer, W.J. Llope, T. Ludlam, R.D. Majka, D. Mackowiecki, S.K. Mark, J.T. Mitchell, M. Muthuswamy, E. O'Brien, L. Olsen, V. Polychronakos, C. Pruneau, M. Rawool-sullivan, F.S. Rontondo, J. Sandweiss, B. Shivakumar, J. Simon, U. Sonnarara, J. Stachel, J. Sullivan, J. Sunier, H. Takai, T. Throwe, H. Van Hecke, L. Waters, K.L. Wolf, D. Wolfe, and c. Woody, Nucl. Phys. 1525 91c(1991). 
Results on the Production of Exotic objects in Relativistic Nucleus-Nucleus Collisions, J. Barrette, R. Bellwied, P. Braun-Munzinger, W.E. Cleland, G. David, M. Fatyga, D. Fox, S.V. Green, J.R. Hall, R. Heifetz, T. Hemmick, N. Herrmann, R. Hogue, G. Ingold, K. Jayanada, D. Kraus, D. Lissauer, W.J. Llope, T. Ludlam, R.D. Majka, D. Mackowiecki, S.K. Mark, J.T. Mitchell, M. Muthuswamy, E. o'Brien, L. Olsen, V. Polychronakos, C. Pruneau, M. Rawool-Sullivan, F.S. Rontondo, J. Sandweiss, B. Shivakumar, J. Simon, U. Sonnarara, J. Stachel, J. Sullivan, J. Sunier, H. Takai, T. Throwe, H. Van Hecke, I. Waters, K.L. Wolf, D. Wolfe, and C. Woody, Nucl. Phys. $\underline{\mathbf{2} 25}$ $369 c(1991)$.

The Design and Construction of a Lead/Scintillator Sampling calorimeter with Fiber optic Readout, J.Simon-Gillo, A. Farooq, M.W. Rawool-sullivan, A. Ray, J. Shoemaker, J.P. Sullivan, K.I. Wolf, E.F. Barasch, J.G. Bossevain, D. Fox, A. Gavron, K. Holzscheiter, B.V. Jacak, T. Lopez, W. Sondheim, J.W. Sunier, H. Van Hecke and B. Wolf, NIM A525 677 (1991).

Forward Baryons in Relativistic Nucleus-Nucleus Collisions, J Barrette, R. Bellwied, P. Braun-Munzinger, W.E. Cleland, G. David, J. Dee, M. Fatyga, D. Fox, S.V. Green, J. Hall, T.K. Hemmick, R. Heifetz, N. Hermann, R.W. Hogue, G. Ingold, K. Jayanada, D. Kraus, B. Shiva Kumar, M. Lisa, D. Lissauer, W.J. Llope, T. Ludlam, R. Majka, D. Mackowiecki, S.K. Mark, J.T. Mitchell, M. Muthuswamy, E. o'Brien, V. Polychronakos, C. Puruneau, F. Rontondo, J. Sandweiss, J. Simon, U. Sonnarara, J, Stachel, H. Takai, T. Throwe, L. Waters, C. Winter, C. Woody, K. Wolf, D. Wolfe, and Y. Zhang, Phys. Rev. C45 $819(1992)$.

Response of the Participant Calorimeter to $1.5-6.8 \mathrm{GeV} / \mathrm{C}$ Electrons and Hadrons, D.Fox, J. Simon-Gillo, M.W. RawoolSullivan, J.G. Boissevain, W.E. Cleland, A. Gavron, B.V. Jacak, D. Kraus, W. Sondheim, J.P. Sullivan, H. van Hecke, S. Watson, K.L. Wolf, and Z. Zhang, NIM A317 474 (1992).

Electromagnetic Dissociation, of Relativistic ${ }^{28} \mathrm{Si}$ into $\mathrm{p}+{ }^{27} \mathrm{Al}$, J. Barrette, R. Bellwied, P. Braun-Munzinger, W.E. Cleland, G. David, J. Dee, O. Dietzsch, E. Deuk, M. Fatyga, D. Fox, S.V. Green, J.R. Hall, T.K. Hemmick, N. Herrmann, R.W. 
Hogue, B. Hong, K. Jayanada, D. Kraus, B. Shiva Kumar, R. Lacasse, D. Lissauer, W.J. Llope, T. Ludlam, R. Majka, D. Mackowiecki, S.K. Mark, S. Mccorkle, J.T. Mitchell, M. Muthuswamy, E. o'Brien, V. Polychronakos, C. Puruneau, F.s. Rontondo, J. Sandweiss, J. Simon-Gillo, U. Sonnarara, J. Stachel, H. Takai, E.M. Takagui, T.G. Throwe, L. Waters, W.J. Willis, C. Winter, K. Wolf, D. Wolfe, C.L. Woody, N. Xu, Y. Zhang, Z. Zhang, and Z. Zou, Phys. Rev. C45 2427(1992).

Charged particle Multiplicity in $28 \mathrm{Si}+\mathrm{Al}, \mathrm{Cu}$, and $\mathrm{Pb}$ Reactions at $\mathrm{E}_{\mathrm{lab}}=14.6 \mathrm{GeV} /$ nucleon, J. Barrette, R. Bellwied, P. Braun-Munzinger, W.E. Cleland, G. David, O. Dietzsch, E. Deuk, M. Fatyga, D. Fox, S.V. Green, J.R. Hall, R. Heifetz, T.K. Hemmick, M. Herman, N. Herrmann, R.W. Hogue, G. Ingold, K. Jayanada, D. Kraus, B. Shiva Kumar, R. Lacasse, D. Lissauer, W.J. Llope, T. Ludlam, R. Majka, D. Mackowiecki, S.K. Mark, S. McCorkle, J.T. Mitchell, M. Muthuswamy, E. O'Brien, L. Olsen, V. Polychronakos, C. Puruneau, M. Rawool-Sullivan, F.S. Rontondo, J. Sandweiss, J. Simon-Gillo, U. Sonnarara, J. Stachel, J.P. Sullivan, J. Sunier, H. Takai, E.M. Takagui, T. Throwe, H. van Hecke, I. Waters, K. Wolf, D. Wolfe, C.L. Woody, N. $X u$, and $Z$. Zhang, Phys. Rev. C46 312(1992).

Recent Results from Experiment E814 at Brookhaven, J. Barrette, R. Bellwied, P. Braun-Munzinger, W.E. Cleland, T.M. Cromier, G. David, J. Dee, G.E. Diebold, O. Dietzsch, M. Fatyga, D. Fox, J.V. Germani, M. Ghalambor-Dezfuli, S. Gilbert, S.V. Green, J.R. Hall, T.K. Hemmick, N. Herrmann, B. Hong, G. Ingold, K. Jayanada, D. Kraus, B. Shiva Kumar, R. Lacasse, D. Lissauer, W.J. Llope, T. Ludlam, R. Majka, D. Mackowiecki, S.K. Mark, S. McCorkle, J.T. Mitchell, M. Muthuswamy, E. O'Brien, L. Olsen, V. Polychronakos, C. Puruneau, F.S. Rontondo, J. Sandweiss, N.C. desilva, J. Simon-Gillo, U. Sonnarara, J. Stachel, H. Takai, E.M. Takagui, T. G. Throwe, C. Winter, G. Wang, L. Waters, K. Wolf, D. Wolfe, C.I. Woody, N. Xu, Y. Zhang, Z. Zhang, and C. Zou, Nucl. Phys. \$544 137 (1992).

Momentum Distributions of Light Mass Fragments in Si-Nucleus Collisions at $14.6 \mathrm{GeV} /$ Nucleon, J. Barrette, R. Bellwied, P. Braun-Munzinger, W.E. Cleland, T. Cromier, G. David, J. Dee, G.E. Diebold, O. Dietzsch, E. Duek, M. Fatyga, D. Fox, S.V. Green, J.R. Hall, T.K. Hemmick, N. Herrmann, B. Hong, G. Ingold, K. Jayanada, D. Kraus, B. Shiva Kumar, R. 
Lacasse, D. Lissauer, W.J. Llope, T. Ludlam, R. Majka, D. Mackowiecki, S.K. Mark, S. McCorkle, J.T. Mitchell, M. Muthuswamy, E. O'Brien, V. Polychronakos, C. Puruneau, F.S. Rontondo, J. Sandweiss, N.C. da Silva, J. Simon-Gillo, J. Slaughter, U. Sonnarara, J. Stachel, H. Takai, E.M. Takagui, T. G. Throwe, C. Winter, L. Waters, K. Wolf, D. Wolfe, C.L. Woody, N. Xu, Y. Zhang, Z. Zhang, and C. Zou, Nucl. Phys. A544 423 (1992).

Antiproton Production in ${ }^{28} \mathrm{Si-Nucleus} \mathrm{Interactions,} \mathrm{J.}$ Barrette, R. Bellwied, P. Braun-Munzinger, W.E. Cleland, T. Cromier, G. David, J. Dee, G.E. Diebold, O. Dietzsch, E. Duek, M. Fatyga, D. Fox, S.V. Green, J.R. Hall, T.K. Hemmick, N. Herrmann, B. Hong, G. Ingold, K. Jayanada, D. Kraus, B. Shiva Kumar, R. Lacasse, D. Lissauer, W.J. Llope, T. Ludlam, R. Majka, D. Mackowiecki, S.K. Mark, S. McCorkle, J.T. Mitchell, M. Muthuswamy, E. O'Brien, V. polychronakos, C. puruneau, F.S. Rontondo, J.' Sandweiss, N.C. da silva, J. Simon-Gillo, J. Slaughter, U. Sonnarara, J. Stachel, H. Takai, E.M. Takagui, T. G. Throwe, C. Winter, L. Waters, K. Wolf, D. Wolfe, C.L. Woody, N. Xu, Y. Zhang, Z. Zhang, and C. Zou, Nucl. Phys. A544 599(1992).

Response of the Participant Calorimeter to Low Energy Pions, Muons, and Positrons, J.P. Sullivan, M.W. RawoolSullivan, J.G. Boissevain, D. Fox, A. Gavron, K. Holzscheiter, B.V. Jacak, J. Simon-Gillo, W.E. Sondheim, H. van Hecke, B. Wolf and K. Wolf, NIM A324 441(1993).

Two-Pion Correlations and Multiplicity Effects in La on La Collisions, H. Bossy, J.A. Bistirlich, R.R. Bossingham, A.D. Chacon, K.M. Crowe, Y. Dardenne, M. Justice, J.O. Rasmussen, A.A. Shihab-Eldin, M.A. Stoyer, K.D. Wyatt, J.P. Sullivan, K.L. Wolf, Phys. Rev. C 471659 (1993).

Antiproton Production in Relativistic Si-Nucleus Collisions, J. Barrette, R. Bellwied, P. Braun-Munzinger, W.E. Cleland, T. Cromier, G. Dadusc, G. David, J. Dee, G.E. Diebold, 0. Dietzsch, E. Duek, M. Fatyga, D. Fox, S.V. Greene, J.V. Germani, J.R. Hall, T.K. Hemmick, N. Herrman, R.W. Hogue, B. Hong, K. Jayananda, D. Kraus, B.S. Kumar, R. Lacasse, D. Lissauer, W.J. Llope, T.W. Ludlam, R. Majka, D. Makowieki, S.K. Mark, J. T. Mitchell, M. Muthuswamy, E. O'Brien, C. Purneau, F.S. Rotondo, J. Sandweiss, N.C. da Silva, J. SimonGillo, J. Slaughter, U. Sonnadara, J. Stachel, H. Takai, E.M. 
Takagui, T.G. Throwe, L. Waters, Ch. Winter, K. Wolf, D. Wolfe, C.L. Woody, N. Xu, Y. Zhang, and C. Zou, Phys. Rev. Lett. 701763 (1993).

Measurement of Transverse Energy Production with $\mathrm{Si}$ and $\mathrm{Au}$ Beams at Relativistic Energy: Towards Hot and Dense Hadronic Matter, J. Barrette, R. Bellwied, S. Bennett, P. BraunMunzinger, W.E. Cleland, T. M. Cromier, G. David, J. Dee, G.E. Diebold, O. Dietzsch, J.E. Diebold, O. Dietzsch, D. Fox, S.V. Greene, J.V. Germani, S. Gilbert, J.R. Hall, T.K. Hemmick, N. Herrman, B. Hong, K. Jayananda, D. Kraus, B. Shiva Kumar, R. Lacasse, Q. Li, D. Lissauer, W.J. Llope, T.W. Ludlam, S. Mccorkle, R. Majka, D. Makowieki, S.K. Mark, R. Matheus, J.T. Mitchell, M. Muthuswamy, E. O'Brien, S. Panitkin, C. Purneau, M.N. Rao, M. Rosati, F. Rotondo, N.C. dasilva, J. Simon-Gillo, U. Sonnadara, J. Stachel, J. Sullivan, H. Takai, E.M. Takagui, T.G. Throwe, C. Winter, G. Wang, K.L. Wolf, D. Wolfe, C.L. Woody, N. Xu, Y. Zhang, Z. Zhang, and C. Zou, Phys. Rev. 70 2996(1993).

Mass and Charge Distributions of Cl-Induced Heavy-Ion Reactions, A.A. Marchetti, A.C. Mignerey, H. Madani, A. Gokmen, W.L. Kehoe, B. Libby, K. Morley, H. Breuer, K. Wolf, F. Obenshain, Phys. Rev. C $\underline{48}$ 266(1993).

Identified Particle Interferometry in Heavy-Ion Collisions:Results From the CERN NA44 Experiment, H. Beker, H. Boggild, J. Boissevain, M. Cherney, J. Dodd, S. Esumi, C.W. Fabjan, D.E. Fields, A. Franz, K.H. Hansen, B. Holzer, T. Humanic, B. Jacak, R. Jayanti, H. Kalechofsky, T. Kobayashi, R. Kvatadze, Y.Y. Lee, M. Leltchouk, B. Lorstad, N. Maeda, A. Medvedev, Y. Miake, A. Miyabayashi, M. Murray, S. Nagamiya, S. Nishimura, E. Noteboom, S.U. Pandey, F. Piuz, V. Polychronakos, M. Potekhin, G. Poulard, A. Sakaguchi, M. Sarabura, K. Shigaki, J. simon-Gillo, H. sletten, W. Sondheim, T. Sugitate, J.P. Sullivan, Y. Sumi, H. van Hecke, W.J. Willis, K. Wolf, Nucl. Phys. A $\underline{566} 115 c(1994)$.

Single Particle Spectra From NA44 H. Boggild, J. Boissevain, M. Cherney, J. Dodd, S. Esumi, C.W. Fabjan, D.E. Fields, A. Franz, K.H. Hansen, B. Holzer, T. Humanic, B. Jacak, R. Jayanti, H. Kalechofsky, T. Kobayashi, R. Kvatadze, Y.Y. Lee, M. Leltchouk, B. Lorstad, N. Maeda, A. Medvedev, Y. Miake, A. Miyabayashi, M. Murray, S. Nagamiya, S. Nishimura, E. Noteboom, S.U. Pandey, F. Piuz, V. Polychronakos, M. 
Potekhin, G. Poulard, A. Sakaguchi, M. Sarabura, K. Shigaki, J. Simon-Gillo, H. Sletten, W. Sondheim, T. Sugitate, J.P. Sullivan, Y. Sumi, H. van Hecke, W.J. Willis, K. Wolf, Nucl. Phys. A 566 515c(1994).

The STAR Experiment at the Relativistic heavy Ion collider, the STAR Collaboration, Nucl. Phys. A $\underline{566} 277$ (1994).

Kaon Interferometry in Heavy-Ion Collisions at the CERN SPS, H. Beker, H. Boggild, J. Boissevain, M. Cherney, J. Dodd, S. Esumi, C.W. Fabjan, D.E. Fields, A. Franz, K.H. Hansen, B. Holzer, T. Humanic, B. Jacak, R. Jayanti, H. Kalechofsky, T. Kobayashi, R. Kvatadze, Y.Y. Lee, M. Leltchouk, B. Lorstad, N. Maeda, A. Medvedev, Y. Miake, A. Miyabayashi, M. Murray, S. Nagamiya, S. Nishimura, E. Noteboom, S.U. Pandey, F. Piuz, V. Polychronakos, M. Potekhin, G. Poulard, A. Sakaguchi, M. Sarabura, K. Shigaki, J. Simon-Gillo, H. Sletten, W. Sondheim, T. Sugitate, J.P. Sullivan, Y. Sumi, H. van Hecke, W.J. Willis, K. Wolf, Z. Phys. C 64 209(1994).

Determination of Critical Exponents from the Multifragmentation of Gold Nuclei, M.L. Gilkes, S. Albergo, F. Bieser, F.P. Brady, Z. Caccia, D.A. Cebra, A.D. Chacon, J.L. Chance, Y. Choi, S. Costa, J.B. Elliot, J.A. Hauger, A.S. Hirsch, E.L. Hjort, A. Insolia, M. Justice, D. Keane, J.C. Kintner, V. Lindenstruth, M.A. Lisa, U. Lynen,.H.S. Mathis, M. McMahan, C. McParland, W.F. Muller, D.L. Olson, M.D. Partlan, N.T. Porile, R. Potenza, G. Rai, J. Rasmussen, H.G. Ritter, J. Romanski, J.L. Romero, G.V. Russo,.H. Sann, R. Scharenberg, A. Scott, $Y$. Shao, B.K. Srivastava, T.J.M. Symons, M. Tincknell, C. Tuve, S. Wang, P. Warren, H.H. Wiemanand K. Wolf Phys. Rev. Lett. $\underline{73}$ 1590(1994).

Use of Aerogel for Imaging Cherenkov Counters, D.E. Fields, H. van Hecke, J. Boissevain,B.V. Jacak, W.E. Sondheim, J.P. Sullivan, W.J. Willis, K. Wolf, E. Noteboom, P.M. Peters and R, Burke, NIM A $\underline{349} 431$ (1994). 